\title{
Effects of the interaction on the properties of spiral galaxies. I. The data ${ }^{\star}$
}

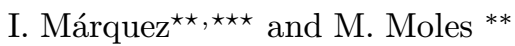 \\ Instituto de Astrofísica de Andalucía (C.S.I.C.), Apdo. 3004, 18080 Granada, Spain
}

Received January 4; accepted March 19, 1996

\begin{abstract}
We have obtained Johnson BVI images and long slit spectra along the major axis of a sample of isolated spiral galaxies, and a sample of spirals in isolated pairs. We present in this contribution the photometric parameters (galactic orientation in the sky, total magnitudes and colors, color gradients, isophotal profiles and their decomposition in bulge and disk components) and the rotation curves of the observed galaxies. For some of the galaxies $\mathrm{H} \alpha$ images and/or spectra along the minor axis are also available and the data also presented here. The data we obtain are then compared with those reported in the RC3 catalogue. The analysis of both samples and their comparison will be published separately.
\end{abstract}

Key words: galaxies: spiral; kinematics and dynamics; photometry; structure; interactions

\section{Introduction}

The properties of an initially non-perturbed galaxy can be modified by gravitational interaction with other systems. These modifications when present, can show up in the morphology, the dynamical properties or the stellar content, depending on the environmental conditions, that is to say, the form and strength of the gravitational interaction.

The peculiarities in the morphology of galaxies in interacting systems are generally explained as due to the effects of tidal forces that can provoke, in the case of spirals, the formation of plumes, tails or bridges together with the triggering of spiral arms or rings (Toomre \& Toomre 1972; Athanassoula 1984). The Atlas of Peculiar Galaxies by Arp (1966) is a clear observational representation of these kind of phenomena. These peculiarities are thought to be the consequence of the modification of the dynamical properties of interacting galaxies (Arkhipova et al.

Send offprint requests to: I. Márquez, marquez@iap.fr

* Based on data obtained at the $1.5 \mathrm{~m}$ telescope of the Estación de Observación de Calar Alto (EOCA), Instituto Geográfico Nacional, which is jointly operated by the Instituto Geográfico Nacional and the Consejo Superior de Investigaciones Científicas through the Instituto de Astrofísica de Andalucía

${ }^{\star \star}$ Visiting Astronomer, German-Spanish Astronomical Center, Calar Alto, operated by the Max-Planck-Institut fur Astronomie jointly with the Spanish National Commission for Astronomy

${ }^{\star \star \star}$ Present Address: Institut d'Astrophysique de Paris, CNRS, 98 bis Bd Arago, F-75014 Paris, France
1986, 1987), affecting both the gaseous and stellar components. Those effects can be reproduced by numerical simulations (Toomre \& Toomre 1972; Noguchi 1987; Barnes \& Hernquist 1992). The stellar content can also be modified as a result of the star formation processes that can be triggered by the gravitational interaction (Larson \& Tinsley 1978; Keel et al. 1985; Moles et al. 1994a and references therein). Indeed, it has been repeatedly observed that the star forming rates are higher in interacting than in isolated galaxies (Keel et al. 1985). On the other hand, a higher percentage of clearly interacting systems (i.e., with perturbed morphologies) is found among galaxies with high star formation rates (Karachentsev \& Karachentseva 1974; Kennicutt \& Kent 1983: Joseph \& Wright 1984; Bushouse 1987; Laurikainen \& Moles 1988).

The effects of the interaction depend on the details of the interaction itself. A puzzle at present is that of the Compact Groups of Galaxies were the star formation rate or the fraction of mergers are considerably smaller than what could be expected (Moles et al. 1994a, and references therein). On the other hand, it has been noticed that some of the properties attributed by default to the effects of the interaction between galaxies can be also found in isolated systems (Campos-Aguilar \& Moles 1991; Campos-Aguilar et al. 1993; Moles et al 1994b). Thus, the identification and analysis of the effects of the gravitational interaction are far from straightforward.

As a first step towards the clarification of all those aspects it seems therefore necessary to characterize the observational properties of isolated galaxies, to establish 
Table 1. Isolated spiral galaxies

\begin{tabular}{l|ccccccccccc}
\hline $\begin{array}{c}\text { Galaxy } \\
(1)\end{array}$ & $\begin{array}{c}m \\
(2)\end{array}$ & $c z$ & $\begin{array}{c}t \\
(3)\end{array}$ & $\begin{array}{c}t(R C 3) \\
(5)\end{array}$ & $\begin{array}{c}B \\
(6)\end{array}$ & $\begin{array}{c}a \\
(7)\end{array}$ & $\begin{array}{c}b \\
(8)\end{array}$ & $\begin{array}{c}E M \\
(9)\end{array}$ & $\begin{array}{c}e m \\
(10)\end{array}$ & $\begin{array}{c}H \alpha \\
(11)\end{array}$ & $\begin{array}{c}B V I \\
(12)\end{array}$ \\
\hline N 214 & 13.0 & 4534 & 5 & SXR5 & 2 & 2.2 & 1.7 & 0 & 0 & 1 & 1 \\
N 718 & 12.8 & 1756 & 1 & SXS1 & 2 & 2.6 & 2.2 & 1 & 0 & 1 & 1 \\
N 818 & 12.7 & 4245 & 5 & SX.5* & 2 & 3.5 & 1.4 & 1 & 0 & 1 & 1 \\
U3511 & 13.0 & 3567 & 6 & S..6* & 0 & 1.6 & 1.2 & 1 & 1 & 1 & 1 \\
U3804 & 13.0 & 2878 & 5 & S..6* & 0 & 1.9 & 1.3 & 1 & 1 & 0 & 1 \\
N2532 & 12.9 & 5251 & 5 & SXT5 & 2 & 2.2 & 1.7 & 1 & 1 & $1^{\prime}$ & 1 \\
N2712 & 12.9 & 1815 & 3 & SBR3 & 3 & 3.5 & 1.7 & 1 & 0 & 1 & 1 \\
I 529 & 12.0 & 2264 & 5 & SAS5 & 1 & 3.9 & 1.8 & 1 & 0 & 0 & 1 \\
N3055 & 13.0 & 1832 & 5 & SXS5 & 2 & 2.2 & 1.2 & 1 & 0 & 0 & 1 \\
N3294 & 12.4 & 1571 & 5 & SAS5 & 1 & 3.8 & 1.8 & 1 & 0 & 0 & 0 \\
N3549 & 12.8 & 2867 & 5 & SAS5 & 1 & 3.3 & 1.0 & 1 & 0 & 0 & 1 \\
N3780 & 12.5 & 2394 & 5 & SAS5 & 1 & 3.1 & 2.5 & 0 & 0 & 0 & 0 \\
N3811 & 13.0 & 3042 & 6 & SBR6* & 3 & 2.5 & 1.6 & 1 & 0 & 0 & 1 \\
N3835 & 13.0 & 2450 & 3 & S..2* & 0 & 2.3 & 0.7 & 1 & 0 & 0 & 1 \\
N4162 & 12.8 & 2571 & 4 & RSAT4 & 1 & 2.5 & 1.3 & 1 & 0 & 0 & 0 \\
N4290 & 12.8 & 3038 & 2 & SBT2 & 3 & 2.5 & 1.9 & 1 & 0 & 0 & 0 \\
N4525 & 13.0 & 1186 & 7 & S...6* & 3 & 3.0 & 1.6 & 1 & 0 & 0 & 0 \\
N5300 & 12.0 & 1179 & 5 & SXR5 & 2 & 3.8 & 2.3 & 0 & 0 & 0 & 0 \\
N5962 & 12.4 & 1963 & 5 & SAR5 & 1 & 2.8 & 2.0 & 1 & 1 & 0 & 1 \\
N6155 & 13.0 & 2418 & 1 & S?.. & 0 & 1.4 & 0.9 & 1 & 1 & 1 & 1 \\
N6395 & 12.8 & 1167 & 5 & S...6* & 0 & 2.7 & 0.8 & 1 & 0 & 0 & 1 \\
N6643 & 11.8 & 1491 & 5 & SAT5 & 1 & 4.0 & 1.9 & 1 & 0 & 1 & 1 \\
\hline
\end{tabular}

(1) Name $\mathrm{N}=\mathrm{NGC}, \mathrm{U}=\mathrm{UGC}, \mathrm{I}=\mathrm{IC}$.

(2) $B$ magnitude (CfA).

(3) Redshift (CfA).

(4) CfA morphological type $(\mathrm{Sa}=1, \mathrm{Sb}=3, \mathrm{Sc}=5, \mathrm{Sd}=7)$.

(5) Morphological type reported in the RC3 catalogue.

(6) CfA bar information ( $3=\mathrm{B}, 2=\mathrm{X}, 1=\mathrm{A}, 0=$ with no information).

(7) CfA major axis diameter in arcminutes.

(8) CfA minor axis diameter in arcminutes.

(9) Long slit spectra along the major axis $(0=$ spectrum not available).

(10) Long slit spectra along the minor axis $(0=$ spectrum not available).

(11) $\mathrm{H} \alpha$ image $\left(0=\right.$ not available, $1^{\prime}=$ continuum not available $)$.

(12) $B V I$ images $(0=$ not available).

quantitatively and qualitatively, what properties are modified by the interaction and in what degree. This is the point we address in the present and following papers. We have selected and observed a sample of isolated spiral galaxies. The parameters we determine will be later used as a zero point to which refer the properties of spiral galaxies in different environments. In particular, we plan to analyze the effects of the gravitational interaction through the comparison of the characteristics of isolated spirals with those found for spirals in isolated pairs. This paper is devoted to the selection of the samples and the description of the data we have obtained. The description of the properties found for each sample and the results on the effects of the interaction will be published separately (Márquez \& Moles, Papers II and III, in preparation). In Sect. 2 we present the procedures for the selection of the samples, in Sect. 3 we present the data, in Sect. 4 we describe the methods of analysis and in Sect. 5 we make a comparison with similar data in the RC3 catalogue.

\section{The selection of the samples}

\subsection{Isolated galaxies}

We started selecting in the CfA catalogue (Huchra et al. 1989) all the spiral galaxies brighter than $m_{B}=13 \mathrm{mag}$ and with $\delta>0$. For practical reasons only galaxies with diameters smaller than $4^{\prime}$ were retained. Since we planned to get both photometric and kinematical data, we only considered objects with inclinations between $32^{\circ}$ and $73^{\circ}$, that is to say, with minor to major axis ratios (as given in the catalogue) in the range $0.8<b / a<0.3$, to minimize the inclination corrections.

Then we defined a criterion for isolation. We first excluded all the galaxies included in the catalogues by Karachentsev (1972), Turner (1976) or Soares (1989), that is to say, well characterized members of isolated pairs or small groups. For the remainder we have considered a galaxy as isolated when all the possible effects of a past interaction, on the morphological or dynamical properties or those concerning the enhancement of star formation processes, have been erased at the present time. Since these 
Table 2. Isolated pairs of spirals

\begin{tabular}{|c|c|c|c|c|c|c|c|c|c|c|c|c|}
\hline $\begin{array}{c}\text { Galaxy } \\
(1)\end{array}$ & $\begin{array}{l}N r \\
(2)\end{array}$ & $\begin{array}{c}m \\
(3)\end{array}$ & $\begin{array}{l}c z \\
(4)\end{array}$ & $\begin{array}{c}t \\
(5)\end{array}$ & $\begin{array}{c}t(R C 3) \\
(6)\end{array}$ & $\begin{array}{c}B \\
(7)\end{array}$ & $\begin{array}{c}a \\
(8)\end{array}$ & $\begin{array}{c}b \\
(9)\end{array}$ & $\begin{array}{l}E M \\
(10)\end{array}$ & $\begin{array}{c}e m \\
(11)\end{array}$ & $\begin{array}{c}\mathrm{H} \alpha \\
(12)\end{array}$ & $\begin{array}{c}B V I \\
(13)\end{array}$ \\
\hline N2341 & 125 & 13.7 & 5219 & 5 & P..... & 0 & 0.9 & 0.9 & 1 & 1 & $1^{\prime}$ & 1 \\
\hline $\mathrm{N} 2342$ & & 12.6 & 5256 & 0 & S...P & 0 & 1.4 & 1.3 & 1 & 1 & $1^{\prime}$ & 1 \\
\hline N2648 & 168 & 13.0 & 1925 & 1 & S..1 & 1 & 3.6 & 1.1 & 1 & 0 & 0 & 1 \\
\hline $0840+1427$ & & 15.0 & 2126 & 9 & $\mathrm{~S} ? \ldots$ & 0 & 0.0 & 0.0 & 1 & 0 & 0 & 1 \\
\hline N2798 & 195 & 13.3 & 1726 & 1 & SB1 & 3 & 2.8 & 0.9 & 1 & 0 & 0 & 0 \\
\hline N2799 & & 14.4 & 1882 & 9 & S... & 3 & 2.1 & 0.5 & 1 & 0 & 0 & 0 \\
\hline N3646 & 281 & 12.1 & 4195 & 4 & RING.. & 0 & 3.8 & 2.2 & 1 & 0 & $1^{\prime \prime}$ & 1 \\
\hline N3649 & & 14.5 & 4442 & 2 & SBS1 & 0 & 1.5 & 0.5 & 0 & 0 & 0 & 1 \\
\hline N3690 & 288 & 12.6 & 3101 & 9 & IB.9P & 3 & 2.9 & 0.0 & 1 & 0 & 1 & 1 \\
\hline I 694 & & 12.6 & 3115 & 9 & SB.9\$P & 3 & 1.1 & 0.9 & 1 & 0 & 1 & 1 \\
\hline N4567 & 347 & 12.4 & 2186 & 4 & S..5 & 1 & 3.0 & 2.5 & 1 & 0 & 0 & 0 \\
\hline N4568 & & 12.0 & 2232 & 4 & S...5 & 1 & 5.1 & 2.4 & 1 & 0 & 0 & 0 \\
\hline N5303A & 397 & 12.9 & 1284 & 5 & P... & 0 & 1.0 & 0.4 & 1 & 0 & 1 & 1 \\
\hline N5303B & & 15.3 & 1428 & 9 & $\mathrm{~S} ? \ldots$ & 0 & 0.0 & 0.0 & 1 & 0 & 1 & 1 \\
\hline N7469 & 575 & 13.1 & 4852 & 1 & PSXT1 & 2 & 1.6 & 1.1 & 1 & 0 & 1 & 1 \\
\hline I5283 & & 15.2 & 4913 & 6 & SAR6P\$ & 1 & 0.0 & 0.0 & 1 & 0 & 1 & 1 \\
\hline N7537 & 578 & 14.1 & 2648 & 4 & SA.\$* & 3 & 2.1 & 0.8 & 1 & 0 & 1 & 1 \\
\hline N7541 & & 12.8 & 2678 & 4 & $\mathrm{SBT} 4 * \mathrm{P}$ & 3 & 3.4 & 1.1 & 1 & 0 & 1 & 1 \\
\hline
\end{tabular}

(1) Name $\mathrm{N}=\mathrm{NGC}, \mathrm{U}=\mathrm{UGC}, \mathrm{I}=\mathrm{IC}$.

(2) Number in Karachentsev's catalogue.

(3) $B$ magnitude (CfA).

(4) Redshift (CfA).

(5) CfA morphological type $(\mathrm{Sa}=1, \mathrm{Sb}=3, \mathrm{Sc}=5, \mathrm{Sd}=7)$.

(6) Morphological type reported in the RC3 catalogue.

(7) CfA bar information ( $3=\mathrm{B}, 2=\mathrm{X}, 1=\mathrm{A}, 0=$ with no information).

(8) CfA major axis diameter $\left({ }^{\prime}\right)$.

(9) CfA minor axis diameter $\left({ }^{\prime}\right)$.

(10) Long slit spectra along the major axis $(0=$ spectrum not available).

(11) Long slit spectra along the minor axis $(0=$ spectrum not available).

(12) $\mathrm{H} \alpha$ image $\left(0=\right.$ not available, $1^{\prime}=$ continuum not available, $1^{\prime \prime}=$ just continuum).

(13) $B V I$ images $(0=$ not available $)$.

effects have a life time of $\approx 10^{9}$ years, we can obtain a condition on the position and redshift of the nearest companion: a galaxy will be considered isolated when the nearest neighbor is placed farther than $0.5 \mathrm{Mpc}$ in projected distance and/or the difference in redshift is greater than $500 \mathrm{~km} \mathrm{~s}^{-1}\left(H_{0}=75 \mathrm{~km} \mathrm{~s}^{-1} \mathrm{Mpc}^{-1}\right)$.

This automatic search could produce some candidates that actually have some companion fainter than the limit of the CfA catalogue, but still very efficient in producing dynamical or morphological perturbations in the main galaxy (Athanassoula 1984). To eliminate those doubtful cases we also used Nilson's (1973) information on the presence of companions and we reexamined the neighborhood of each galaxy in the Palomar Sky Survey Prints. The galaxies with small optical companions were excluded from the final list of isolated galaxies even if in most cases the redshifts of the companions are not known. In one case, that of NGC 6701, the nearby small galaxy resulted to be a physical companion, presumably responsible for the peculiarities found in NGC 6701 (Márquez et al. 1996).

The final sample of isolated galaxies comprises 22 galaxies (see Table 1). The sample selected in that way is magnitude and size limited. All the selected galaxies resulted to be nearby objects $\left(c z<6000 \mathrm{~km} \mathrm{~s}^{-1}\right)$. The morphological subtypes are mostly late spirals (Sc typically). Therefore, and considering the sample number, it will not be possible to statistically describe the properties of every subtype. On the contrary, the sample of isolated spirals will be used to obtain a general characterization of the family as a whole.

\subsection{Isolated pairs of spiral galaxies}

We have selected from the catalogue by Karachentsev (1972, 1980, 1987), all those pairs in which at least one of the members (what we call the primary galaxy) fulfills the same conditions in magnitude, declination, size and inclination as for the sample of isolated galaxies. We have also applied the same isolation criteria to the pair as a whole. The resulting sample of isolated pairs of spiral galaxies contains 9 systems (Table 2). The morphological distribution is rather different than that of isolated spirals, since the primary galaxies among the isolated pairs are mainly early type spirals. We note that this difference will have to be considered as a possible bias when comparing both samples, since the effects of the gravitational 
interaction could depend on the relative contribution of the spheroidal component (see, for instance, Márquez \& Moles 1994).

\section{Observations and data reduction}

We obtained broad band $B V I$ images for 16 out of the 22 selected isolated galaxies, and for 7 out of the 9 selected isolated pairs along 7 observing runs, in Calar Alto (Almería, Spain) and La Palma (Tenerife, Spain) observatories. The general characteristics of the observations are given in Table 3 and the details for each galaxy in Table 5 . The quality of the whole images was determined by fitting a bi-dimensional Moffat's (1969) profile to the projected stars in each frame. The seeing values obtained in that way are also given in Table 5 .

The data were de-biased and flat-fielded using the corresponding standard routines in FIGARO. Standards from Landoldt's (1983) list for the first two runs and from the list by Neckel \& Chini (1980) for the other were observed for extinction and calibration purposes. The photometric data were reduced following Moles \& García-Pelayo (1985). The errors in the standard stars are quoted as quality and given in Table 3 .

To control the accuracy of the target images we proceeded in the following way. We first constructed the colorcolor diagrams $((B-V)$ vs. $(V-I)$ and $(B-V)$ vs. $(B-I))$ for all the observed stars in a given frame. To built up a simple reference we fitted a parabola to the points corresponding to the observed standard stars. The resulting $\mathrm{rms}$ values amount to $\sigma_{B V / V I}^{\mathrm{Standard}}=0.035$, and $\sigma_{B V / B I}^{\mathrm{Standard}}=$ 0.026 , which are similar to the quoted errors for those stars. Then we compared the colors of the observed foreground stars with respect to that fitting parabola, the rms of the differences being a measure of the calibration error in each frame. That comparison indicates a quality similar to that obtained for the standard stars themselves. In Fig. 1 we show the results for the set of images from February 1992, with different symbols for each observed galaxy. It can be seen that the dispersion of foreground stars is well within the range given by the standard stars.

The surface brightness corresponding to the isophote at $3 \sigma$ over the sky level is given as an indicator of the depth we achieved (Table 5).

We could also obtain $\mathrm{H} \alpha$ continuum subtracted images for 7 isolated galaxies and 4 isolated pairs. These narrow band images were obtained during 6 runs with the Spanish $1.52 \mathrm{~m}$ telescope in Calar Alto. The details are given in Tables 1-3 and 5. The calibration was made through the observation of spectrophotometric standard stars from the list by Oke (1974). We used the calibration procedure described in Márquez \& Moles (1993). The accuracy is always better than $5 \%$ and is given in Table 3 .

The spectroscopic data were collected with the Cassegrain Spectrograph attached to the $2.2 \mathrm{~m}$ telescope in Calar Alto, and with the Intermediate Dispersion Spec-
Table 3. Log of the observing runs (images)

\begin{tabular}{|c|c|c|c|c|c|}
\hline Date & Telescope & Band & $\mathrm{CCD}$ & $\begin{array}{c}\text { Scale } \\
\left({ }^{\prime \prime} / \mathrm{px}\right)\end{array}$ & $\begin{array}{l}\text { Phot. } \\
\text { Accur. }\end{array}$ \\
\hline Jul.91 & $\underset{"}{\mathrm{EOCA}}$ & $\begin{array}{l}\text { BVI } \\
\mathrm{H} \alpha\end{array}$ & $\begin{array}{c}\text { Thom. } \\
\text {. }\end{array}$ & $\begin{array}{c}0.31 \\
"\end{array}$ & $\begin{array}{l}2 \% \\
5 \%\end{array}$ \\
\hline Oct.91 & EOCA & $\mathrm{H} \alpha$ & Thom. & 0.31 & $5 \%$ \\
\hline Feb.92 & EOCA & BVI & Thom. & 0.31 & $4 \%$ \\
\hline Mar.92 & EOCA & $\mathrm{H} \alpha$ & Thom. & 0.31 & $5 \%$ \\
\hline Sep.92 & EOCA & BVI & Thom. & 0.31 & $2-3 \%$ \\
\hline Nov.92 & $\underset{"}{\mathrm{EOCA}}$ & $\begin{array}{l}\text { BVI } \\
\mathrm{H} \alpha\end{array}$ & $\begin{array}{c}\text { Thom. } \\
\text {. }\end{array}$ & $\underset{\#}{0.31}$ & $\begin{array}{c}2 \% \\
6-10 \%\end{array}$ \\
\hline Mar.93 & EOCA & $\mathrm{H} \alpha$ & Thom. & 0.31 & $4 \%$ \\
\hline Feb.94 & CAHA & BVI & Tek\#6 & 0.28 & $3 \%$ \\
\hline Apr.94 & JKT & BVI & EEV5 & 0.31 & $2 \%$ \\
\hline
\end{tabular}

Table 4. Log of the observing runs (spectra)

\begin{tabular}{lcccc}
\hline Date & Telescope & $\begin{array}{c}\text { Dispersion } \\
\AA / \mathrm{px}\end{array}$ & $\begin{array}{c}\text { Spectral range } \\
\AA\end{array}$ & $\begin{array}{c}\text { Scale } \\
\text { " } / \mathrm{px}\end{array}$ \\
\hline Jun.91 & CAHA & 1.31 & $5926-7151$ & 1.35 \\
Feb.93 & INT & 0.79 & $6000-7000$ & 0.65 \\
Mar.93 & CAHA & 1.36 & $5736-7138$ & 1.69 \\
Feb.94 & CAHA & 1.31 & $5926-7151$ & 1.41 \\
\hline
\end{tabular}

trograph (I.D.S) at the $2.5 \mathrm{~m}$ Isaac Newton telescope in La Palma. The slit was normally oriented along the galaxy major axis. The projected slit width was $1 . .5$. Only for a few cases we also observed along the minor axis. Similar configurations were used in both telescopes, covering a spectral range of about $1400 \AA$, centered in the $\mathrm{H} \alpha$ line. All the galaxies for which we have broad band photometry were observed. For other 4 isolated galaxies and 2 pairs only the spectroscopic information is available. Finally, there are 2 galaxies (NGC 3780 and NGC 5300) for which we do not have neither images nor spectra.

The characteristics of the spectroscopic observations are given in Table 4 . The data were wavelength calibrated using He-Ar comparison spectra, which were observed before and after each object exposure. The accuracy of the calibration was tested using the sky lines [OI] $\lambda 6300$ and [OI] $\lambda 6364$. To do so, we calculated the position of the centroids of the sky lines for each section. The average value would give us the corresponding zero in velocities (for which each galaxy has been corrected). The rms error for each spectrum, that is to say, the dispersion around the average value, is given in Table 6, together with dates, position angles and exposition times for the different frames. The sky background level was determined taking median averages over two strips at both sides of the galaxy signal.

The kinematical data were obtained through the use of cross-correlation technics (Tonry \& Davis 1979). The spatial section in the $2 \mathrm{D}$ spectrum with the highest $S / N$ ratio was used as a template for the cross-correlation. The 
errors are referred to the determination of the velocity shift with respect to the template spectrum. They are shown as the error bars in all the velocity distributions (see Fig. 2h and Figs. 4 and 5).
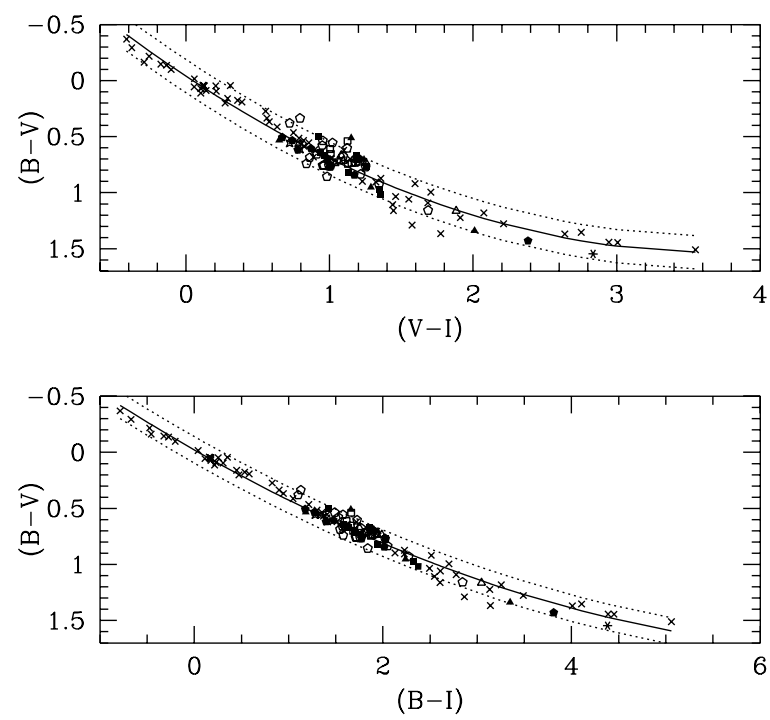

Fig. 1. Color-color plot diagram for the stars observed during the February 1992 run. The position of the standard stars are given by crosses. The thin line corresponds to the average position of the standards, calculated by fitting a parabola to the data. The dashed lines represent the $\pm 2 \sigma$ level from the fit. The other different symbols represent the position of the foreground stars in the frames corresponding to the galaxies observed during that run

\section{Analysis of the data and results}

\subsection{Broad band images}

The main photometric results are presented in Fig. 2. For each galaxy an isophotal image (generally $V$ ) and a greyscale image (generally $B$ ) are shown. These images let us to appreciate important morphological aspects like the bulge contribution, the presence of bars and/or rings and the shape of the spiral arms.

The data displayed in Fig. 2 were used to calculate the total magnitudes and color indices of the galaxies. We used circular apertures to compute the flux at a given equivalent radius and the resulting curve of growth to determine total magnitudes and colors. The errors were estimated taking into account the statistical noise (using poissonian statistics) and the data sampling. For the last one, the error corresponds to the difference between the expected continuous value and the discrete observed one, and it is parametrized as the rms of the difference between a given observed point and the polinome $(n=3)$ fitted to the 4 nearest points to it. These errors resulted to be $\approx 0.06$ mag. Considering the calibration accuracy, we can safely conclude that the total errors are always smaller than 0.1 magnitudes.

The parameters describing the orientation of the galaxy in the plane of the sky were normally calculated by fitting an ellipse to the faintest isophote with enough $S / N$ ratio to be clearly distinguished from the noise level (at $3 \sigma$ in most cases). In the cases where the shape of the outer isophotes was irregular, the ellipticity and position angle were measured on the sharp-divided (original divided by a smoothed, see below) image (NGC 2532 , NGC 2341 and NGC 2342).

These parameters are given in Table 7 for the two samples considered here. The colors and color indices corrected for galactic extinction (after Burstein \& Heiles 1984), internal extinction and $\mathrm{K}$ effect (following the recipes in the $\mathrm{RC} 3$ ) are also given.

In addition to the global information given by the total colors, we used the combination of the bidimensional data in the different bands to obtain a more detailed characterization of the radial changes in the colors (whose interpretation and implications will be given in Papers II and III). The color gradients (also calculated through circular apertures), are shown in Fig. 3. It can be seen that they generally show a roughly linear part, reaching the outskirts. We have fitted it by a straight line ir order to proceed with an analysis of the gradients in spiral galaxies in the two samples. The analysis of the values for the origin and the slope of the fits will be given and discussed elsewhere, together with a comparison of the results for isolated and interacting galaxies (Paper III).

We also show a greyscale color index image (in general, $(B-V)$ ), of each galaxy. This is specially useful to visualize the stellar population distribution and its relation with the different morphological sub-structures. The bulge population is characterized by colors that are redder than those for the disk, over which the spiral arms are traced, with bluer colors. Extremely red colors would imply the presence of dust, a very important component in the interpretation and/or decomposition procedure of the surface brightness profiles, as we will see below.

In order to enhance special features, we have applied contrast-enhancing techniques, as those described by Sofue (1993). The original image is divided by that obtained by convolution with a gaussian with $\mathrm{HPBW}=10$ pixels. This technique allows the subtraction of the diffuse background in a very convenient way to discuss the possible presence of both dust extinguished and very luminous regions (Sofue et al. 1994). The divided, so called sharp divided image for each galaxy is also displayed in Fig. 2.

The usual way of obtaining photometric profiles is based on integration in elliptical strips and ellipse fitting. This method becomes somewhat problematic for strongly non-elliptical isophotes, as it is normally found in the 
Table 5. Detailed log of the images

\begin{tabular}{|c|c|c|c|c|c|}
\hline Galaxy & Band & Date & FWHM & Time(sec) & Faintest isophote \\
\hline \multirow[t]{2}{*}{ NGC 214} & $B V I$ & $11 / 26 / 92$ & 1.1 & $2800,1500,1400$ & $23.8,23.9,21.5$ \\
\hline & $\mathrm{H} \alpha$ & $11 / 27 / 92$ & 1.1 & 2100,2100 & \\
\hline \multirow[t]{2}{*}{ NGC 718} & $B V I$ & $02 / 04 / 92$ & 1.5 & $1800,1200,1000$ & $22.9,22.9,20.2$ \\
\hline & $\mathrm{H} \alpha$ & $11 / 30 / 92$ & - & 2100,2100 & \\
\hline \multirow[t]{2}{*}{ NGC 818} & $B V I$ & $02 / 02 / 92$ & 2.7 & $1800,1200,1000$ & $22.8,23.6,21.6$ \\
\hline & $\mathrm{H} \alpha$ & $11 / 27 / 92$ & 1.3 & 2100,2100 & \\
\hline \multirow[t]{2}{*}{ UGC 3511} & $B I$ & $02 / 03 / 92$ & 1.5 & $1800,-, 1000$ & $23.6,-, 21.6$ \\
\hline & $\mathrm{H} \alpha$ & $11 / 27 / 92$ & 1.3 & 2100,2100 & \\
\hline \multirow[t]{2}{*}{ UGC 3804} & $B V I$ & $02 / 04 / 92$ & 1.1 & $1800,1200,1000$ & $22.7,23.5,22.7$ \\
\hline & $\mathrm{H} \alpha$ & $03 / 20 / 93$ & 1.5 & 2100,2100 & \\
\hline \multirow[t]{2}{*}{ NGC 2532} & $B V I$ & $02 / 01 / 92$ & 2.5 & $1800,1200,1000$ & $23.7,23.2,21.5$ \\
\hline & $\mathrm{H} \alpha$ & $11 / 27 / 92$ & - & 1400 & \\
\hline \multirow[t]{2}{*}{ NGC 2712} & $B V I$ & $02 / 02 / 92$ & 2.5 & $1800,1200,1000$ & $23.5,23.4,21.8$ \\
\hline & $\mathrm{H} \alpha$ & $03 / 24 / 93$ & 1.5 & 2100,2100 & \\
\hline IC 529 & $B V I *$ & $04 / 10 / 94$ & 1.0 & $2800,2000,1600$ & $23.9,24.0,22.0$ \\
\hline NGC 3055 & $B V I *$ & $04 / 11 / 94$ & 1.5 & $2800,2000,1600$ & $24.2,24.1,22.1$ \\
\hline NGC 3549 & $B V I * *$ & $02 / 15 / 94$ & 4 & $2800,2100,1400$ & $24.7,24.6,23.4$ \\
\hline NGC 3811 & $B V I *$ & $04 / 09 / 94$ & 1.0 & $2800,2000,1600$ & $23.6,23.7,21.6$ \\
\hline NGC 3835 & $B V I *$ & $04 / 11 / 94$ & 1.0 & $2800,2000,1600$ & $24.2,24.3,22.3$ \\
\hline NGC 5962 & $B V I *$ & $04 / 09 / 94$ & 1.0 & $2800,2000,1600$ & $23.7,23.7,21.5$ \\
\hline \multirow[t]{2}{*}{ NGC 6155} & $B V I$ & $07 / 14 / 91$ & 1.1 & $1600,1600,1000$ & $23.0,22.7,23.0$ \\
\hline & $\mathrm{H} \alpha$ & $07 / 13 / 91$ & 1.4 & 1200,1500 & \\
\hline NGC 6395 & $I$ & $07 / 15 / 91$ & 2 & 1000 & $-, 22.7,22.5$ \\
\hline \multirow[t]{2}{*}{ NGC 6643} & $B V I$ & $07 / 14 / 91$ & 1.5 & $1400,1200,1000$ & $23.2,22.8,22.5$ \\
\hline & $\mathrm{H} \alpha$ & $07 / 12 / 91$ & 1.2 & 1500,1500 & \\
\hline \multirow[t]{2}{*}{ Kara 125} & $B V I$ & $02 / 03 / 92$ & 2.3 & $1800,1200,1000$ & $23.3,22.8,21.0$ \\
\hline & $\mathrm{H} \alpha$ & $03 / 22 / 93$ & 1.2 & 2100 & \\
\hline Kara 168 & $B V I$ & $02 / 01 / 92$ & 2.3 & $1800,1200,1000$ & $23.1,22.8,20.7$ \\
\hline \multirow[t]{2}{*}{ Kara 281a } & $B V$ & $02 / 01 / 92$ & 2.0 & 1600,1000 & $23.1,23.8,-$ \\
\hline & $\mathrm{H} \alpha$ & $03 / 21 / 93$ & 1.9 & 2100 & \\
\hline Kara 281b & $B I$ & $02 / 02 / 92$ & 2.5 & $1800,-, 1000$ & $23.7,-, 21.3$ \\
\hline \multirow[t]{2}{*}{ Kara 288} & $B I$ & $02 / 04 / 92$ & 1.2 & $1800,-, 1000$ & $23.4,23.4,20.8$ \\
\hline & $\mathrm{H} \alpha$ & $02 / 28 / 92$ & 1.7 & 2000,2000 & \\
\hline \multirow[t]{2}{*}{ Kara 397} & $B V I$ & $07 / 14 / 91$ & 1.4 & $1800,1200,1000$ & $22.9,23.1,22.5$ \\
\hline & $\mathrm{H} \alpha$ & $07 / 13 / 91$ & 1.4 & 1410,2000 & \\
\hline \multirow[t]{2}{*}{ Kara 575} & $B V I$ & $09 / 03 / 92$ & 1.2 & $1800,1500,990$ & $23.1,23.2,21.1$ \\
\hline & $\mathrm{H} \alpha$ & $10 / 02 / 91$ & 3 & 2000,2000 & \\
\hline \multirow[t]{2}{*}{ Kara 578} & $B V I$ & $09 / 05 / 92$ & 1.0 & $2100,1500,990$ & $23.4,23.11,20.9$ \\
\hline & $\mathrm{H} \alpha$ & $07 / 12 / 91$ & 1.5 & 1800,1800 & \\
\hline
\end{tabular}

* JKT telescope, ${ }^{* *} 2.2$ m CAHA telescope; otherwise, EOCA telescope.

regions crossed by prominent spiral arms. To avoid this problem, we have derived the profiles directly, plotting the isophotal levels versus their equivalent radii, calculated from the area inside each observed isophote. The profiles are displayed in Figs. 2f and 3. We have followed the procedure described by Boroson (1981) to get the photometric components. First, the most external parts of a given profile were used to determine the disk (the so-called "marking the disk" method). We used an exponential law of the form (Freeman 1970),

$$
\log \left(I_{D}\right)=\log \left(I_{D o}\right)-0.7290\left(r / r_{D}-1\right)
$$

The fitted disk was then subtracted from the whole profile and considered as the bulge. A $r^{1 / 4}$, de Vaucouleurs (1948) law of the form

$$
\log \left(I_{B}\right)=\log \left(I_{B o}\right)-3.3307\left(\left(r / r_{B}\right)^{1 / 4}-1\right) .
$$

was used. The bulge was then subtracted from the profile to redetermine the disk. After a few iterations the solution converges to the values finally adopted for the disk and bulge components. The values so obtained are given in Tables 8 and 9. The minimum radius for the disk fitting is also given. In Fig. 3 we plot the differences between observed and fitted profiles for the galaxies in both samples.

The photometric decomposition is not always without problems. Thus, it is rather difficult to extract the pure disk component in early spirals, due to the fact that the bulge contribution is not negligible even towards the outskirts of the detected light distribution. It is neither feasible for those profiles for which the intrapolated disk component would give a central luminosity greater than that 

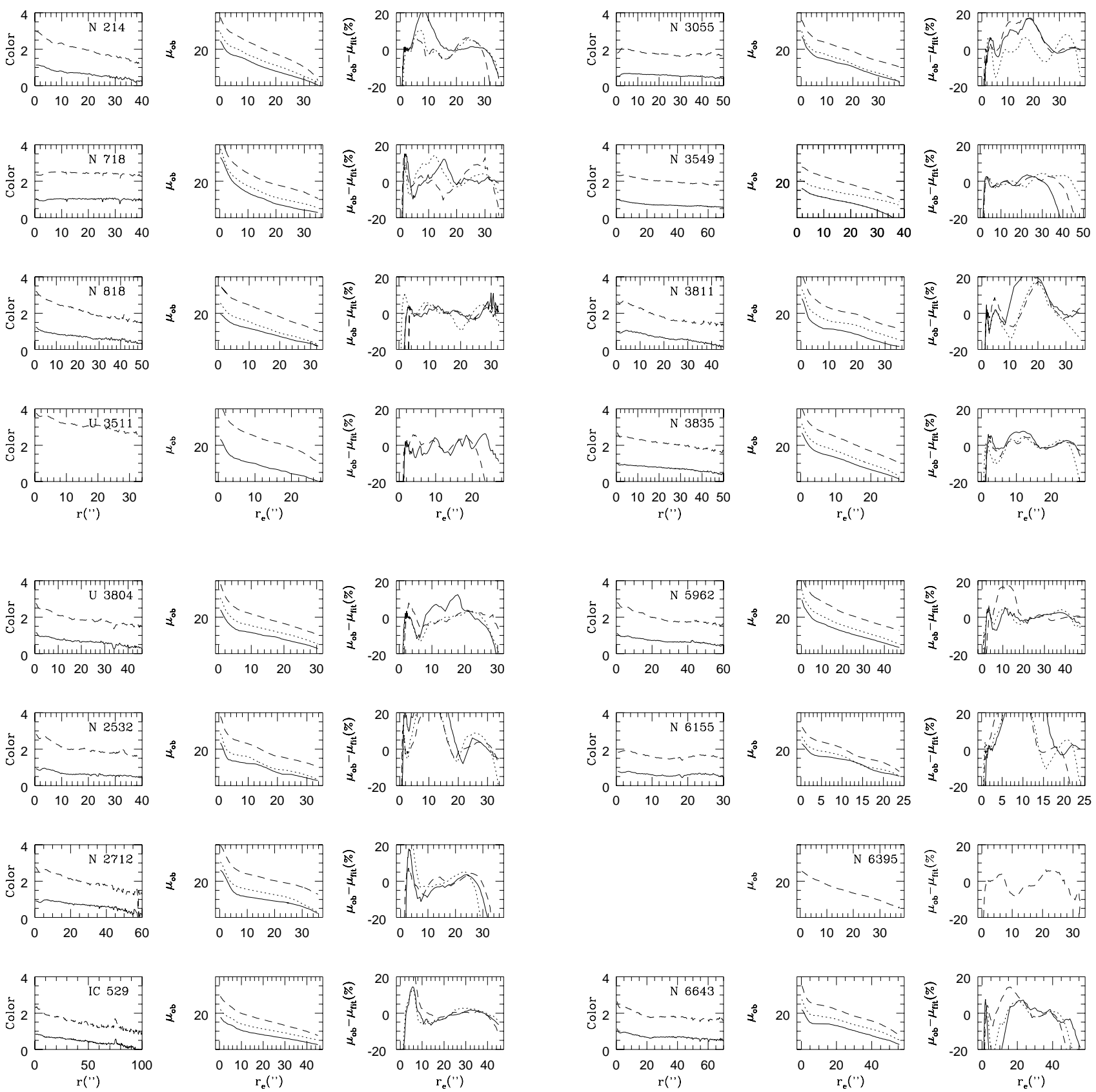

Fig. 3. The plots in each row correspond, from left to right, to a) the color gradients $(B-V)$ (solid line) and $(B-I)($ dashed line), b) the surface brightness profiles in $B$ (solid line), $V$ (dotted line) and $I$ (dashed line) bands, and c) the differences (\%) between observed and fitted profiles (symbols as in $\mathbf{b}$ ))

actually observed (type II profiles, Freeman 1970). The galaxies for which the photometric decomposition suffers from these problems are indicated in Appendix A. It is to note that the comparison of the $B / D$ ratios with those derived in previous works is not very much affected by the different procedure of profile determination; in fact, it has been already proved that the results obtained by different authors, even using the same decomposition method, show a rather large range (Knapen \& van der Kruit 1991).

For the cases were the bulge-disk decomposition can be worked out acceptably, that information can be used together with the orientation parameters to create a simulated image. This image can be then subtracted from the original to trace features like spiral arms, bars, lens or rings, and those components that are not well fitted 

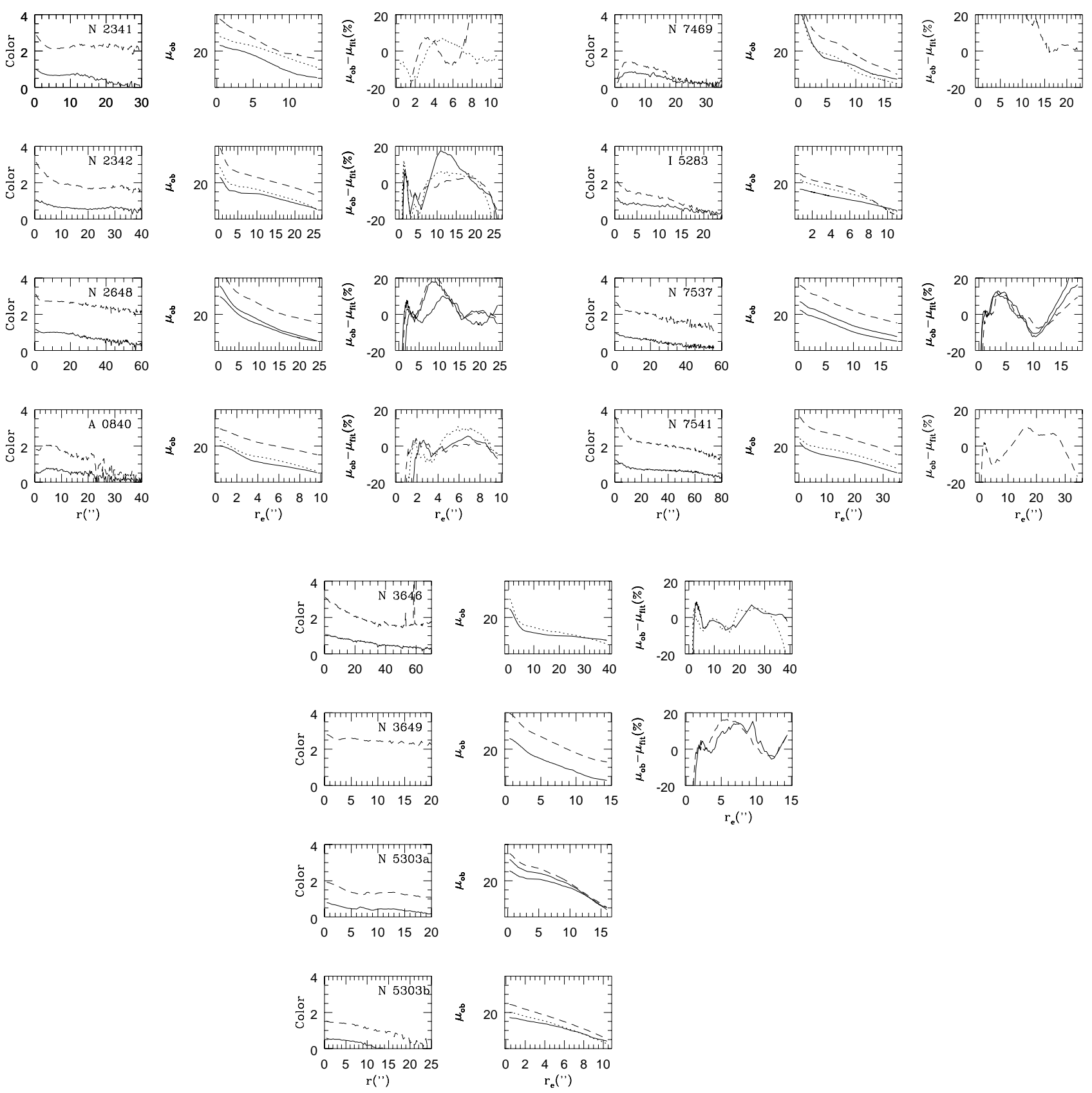

Fig. 3. continued

by neither the exponential nor the de Vaucouleurs laws. The method is particularly well suited to enhance features placed rather near to the center, that could hardly be seen otherwise. These details can be used for a finer analysis of the morphology as compared to previously reported data. It is clear that those techniques are particularly useful when studying the presence of non-axisymmetric components in isolated spiral galaxies, a point that could be of great relevance to elucidate the conditions for such galaxies to develop an active nucleus (Moles et al. 1995).

The results for the individual galaxies are briefly presented in Appendix A, whereas the global analysis and discussion will be included in a forthcoming article.

We have used Fourier analysis of the de-projected images to quantify the importance and aspect of the non-axisymmetric components (Schweizer 1976; Puerari \& Dottori 1992). Given $I(r, \theta)$, the observed intensity 
Table 6. Detailed $\log$ of the spectra

\begin{tabular}{|c|c|c|c|c|}
\hline Galaxy & Date & $\begin{array}{l}\text { PA } \\
\left({ }^{\circ}\right)\end{array}$ & $\begin{array}{l}\text { Time } \\
(\mathrm{sec})\end{array}$ & $\begin{array}{c}\mathrm{rms} \\
(\mathrm{km} / \mathrm{s})\end{array}$ \\
\hline NGC 718 & $02 / 12 / 93$ & 45 & 2700 & 1.6 \\
\hline \multirow[t]{2}{*}{ NGC 818} & $02 / 13 / 93$ & 113 & 3600 & 0.6 \\
\hline & $01 / 17 / 94 *$ & 23 & 3000 & 2.7 \\
\hline \multirow[t]{2}{*}{ UGC 3511} & $02 / 15 / 93$ & 135 & 3600 & 1.5 \\
\hline & $02 / 17 / 94 *$ & 45 & 3000 & 2.5 \\
\hline UGC 3804 & $02 / 12 / 93$ & 13 & 3600 & 2.3 \\
\hline \multirow{2}{*}{ NGC 2532} & $02 / 12 / 93$ & 10 & 3600 & 2.0 \\
\hline & $02 / 16 / 93$ & 100 & 3600 & 2.4 \\
\hline NGC 2712 & $02 / 13 / 93$ & 178 & 3600 & 2.7 \\
\hline IC 529 & $03 / 28 / 93^{*}$ & 145 & 3000 & 0.5 \\
\hline NGC 3055 & $02 / 27 / 93^{*}$ & 63 & 3000 & 0.5 \\
\hline NGC 3294 & $03 / 25 / 93^{*}$ & 122 & 3000 & 1 \\
\hline NGC 3549 & $02 / 15 / 93$ & 38 & 3600 & 2.5 \\
\hline NGC 3811 & $02 / 15 / 93$ & 160 & 3600 & 6.6 \\
\hline NGC 3835 & $02 / 16 / 93$ & 60 & 3600 & 4.6 \\
\hline NGC 4162 & $02 / 14 / 93$ & 174 & 3600 & 3 \\
\hline NGC 4290 & 03/29/93* & 90 & 3600 & 1 \\
\hline NGC 4525 & $03 / 27 / 92^{*}$ & 53 & 3000 & 1 \\
\hline \multirow[t]{2}{*}{ NGC 5962} & $06 / 22 / 91^{*}$ & 110 & 1800 & 4.4 \\
\hline & $06 / 22 / 91^{*}$ & 20 & 800 & 4.8 \\
\hline \multirow{2}{*}{ NGC 6155} & $06 / 18 / 91^{*}$ & 145 & 2500 & 5.3 \\
\hline & $06 / 22 / 91^{*}$ & 55 & 1000 & 6.4 \\
\hline NGC 6395 & $06 / 17 / 91^{*}$ & 15 & 2500 & 4.6 \\
\hline NGC 6643 & $06 / 17 / 91^{*}$ & 38 & 2000 & 4.9 \\
\hline \multirow[t]{2}{*}{ NGC 2341} & $02 / 13 / 93$ & 136 & 3600 & 2.5 \\
\hline & $02 / 14 / 93$ & 46 & 3600 & 1.8 \\
\hline \multirow[t]{2}{*}{ NGC 2342} & $02 / 13 / 93$ & 126 & 3600 & 3.7 \\
\hline & $02 / 13 / 93$ & 36 & 3600 & 2.4 \\
\hline NGC 2648 & $03 / 29 / 93^{*}$ & 148 & 3000 & 0.9 \\
\hline A $0840+1427$ & $03 / 29 / 93^{*}$ & 79 & 3000 & 0.4 \\
\hline NGC 2798 & $03 / 30 / 93^{*}$ & 160 & 3000 & 2 \\
\hline NGC 2799 & $03 / 30 / 93^{*}$ & 125 & 3000 & 1 \\
\hline NGC 3646 & $02 / 15 / 93$ & 50 & 3600 & 1.4 \\
\hline NGC 3690 & $02 / 12 / 93$ & 173 & 3300 & 2.4 \\
\hline$"+$ IC 694 & $02 / 12 / 93$ & 80 & 1800 & 1.3 \\
\hline NGC 4567 & $03 / 30 / 93^{*}$ & 85 & 3000 & 1 \\
\hline NGC 4568 & $03 / 30 / 93^{*}$ & 23 & 3000 & 1 \\
\hline NGC 5303a & $06 / 18 / 91^{*}$ & 83 & 1200 & 6.0 \\
\hline NGC $5303 b$ & $06 / 18 / 91^{*}$ & 110 & 2000 & 4.0 \\
\hline NGC 7469 & $06 / 18 / 91^{*}$ & 130 & 2000 & 3.7 \\
\hline IC 5283 & $06 / 13 / 91^{*}$ & 125 & 2800 & 4.2 \\
\hline NGC 7537 & $06 / 19 / 91^{*}$ & 79 & 1800 & 5.7 \\
\hline NGC 7541 & $06 / 20 / 91 *$ & 102 & 1400 & 4.8 \\
\hline
\end{tabular}

* 2.2 m CAHA telescope: otherwise, INT telescope.

at a given radius as a function of the azimuthal angle $(0 \leq \theta \leq 2 \pi)$, its Fourier transformed, $F T(r, m)$, will correspond to the extension and position angle of the non-axisymmetric components of frequency $m$. We have calculated the components $m=1, \ldots, 6$, to be able to interpret their relative distribution in connection with the observed features (Ohta et al. 1990). Particularly useful is the method for well defined features as bars and spiral arms. The resulting information was used together with the results from the masking and simulated images to proceed with a detailed morphological classification to be compared (or added) to that given by the RC3 catalogue (see Sect. 5).

\section{2. $H \alpha$ images}

$\mathrm{H} \alpha$ images obviously produce most valuable information on the present star formation characteristics of a given galaxy. The point is that not only total fluxes are relevant to evaluate the present star formation rate, but even the distribution and size of the HII regions could reveal peculiarities in the way a given object is forming stars. Moreover, in combination with broad band images, the $\mathrm{H} \alpha$ image data are specially useful to trace the star formation history of galaxies (Márquez 1994).

Following the Gallaguer et al. (1984) prescriptions, the evolutionary history of the star formation processes in a galaxy can be globally depicted just comparing the star formation rates at three different epochs, namely the early stages, the period about $10^{9}$ years ago and the present time. These rates are well reflected by such global properties as the total mass, the $B$ luminosity and the total $\mathrm{H} \alpha$ luminosity, respectively. We insist however that when the point to elucidate is whether a galaxy is now in a peculiar phase of star formation, attention should also be payed to the distribution and sizes of the HII regions, since galaxies with $\mathrm{H} \alpha$ total fluxes rather normal could be peculiar in the detailed distribution and properties of the star forming regions (i.e, NGC 7469, see Márquez \& Moles 1994).

In this respect, the comparison of $\mathrm{H} \alpha$ and broad band color images can already lead to an estimation of how the recent star formation history of a galaxy has taken place in different regions (Márquez \& Moles 1994). Indeed, in general, the HII regions would correspond to the areas with the bluest colors, and this is normally the case. But it is sometimes found that rather blue areas do not present $\mathrm{H} \alpha$ emission. This probably indicates that the HII region seating there in the relatively recent past was turned off. The ionizing stars would have had enough time to evolve, loosing their ionizing power, but the population of early type, blue stars would still be important. With this kind of information it is possible to trace a row image of how the star forming processes have developed in the recent past as a function of the position in the galaxy. Such cases will be briefly discussed in Appendix A (see NGC 3690/IC 694, NGC 5303b, IC 5283, NGC 7541).

The quantitative analysis we have performed comprises the determination of the total $\mathrm{H} \alpha$ emission (Table 7), together with the sizes and luminosities of the HII regions. The relative importance of the contribution by the diffuse emission and the difference between the total emission and that from HII regions will be discussed in the forthcoming papers.

\subsection{Spectroscopy}

\subsubsection{Kinematics}

The velocity distribution given by the spectrum along the galactic major axis is used to obtain the rotation curve. We have defined the kinematic center as that section for 
Table 7. Total parameters

\begin{tabular}{|c|c|c|c|c|c|c|c|c|c|c|c|}
\hline \multirow[b]{2}{*}{ Galaxy } & \multicolumn{3}{|c|}{ Observed } & \multicolumn{3}{|c|}{ Corrected } & \multicolumn{4}{|c|}{ Orientation } & \multirow{2}{*}{$\frac{-\log F(\mathrm{H} \alpha)}{\left(\operatorname{erg~cm}^{-2} \mathrm{~s}^{-1}\right)}$} \\
\hline & $m_{B}$ & $(B-V)$ & $(B-I)$ & $m_{B}^{\mathrm{O}}$ & $(B-V)^{0}$ & $(B-I)^{0}$ & $a$ & $b$ & $P A$ & $i$ & \\
\hline NGC 214 & 13.01 & 0.63 & 1.99 & 12.75 & 0.54 & 1.81 & 1.86 & 1.34 & 45 & 44 & 11.46 \\
\hline NGC 718 & 12.54 & 0.90 & 2.30 & 12.39 & 0.85 & 2.19 & 2.2 & 1.75 & 40 & 37 & (1) \\
\hline NGC 818 & 13.10 & 0.62 & 2.13 & 12.36 & 0.42 & 1.67 & 3.5 & 1.4 & 113 & 66 & 11.68 \\
\hline UGC 3511 & 13.75 & - & 3.04 & 13.31 & - & 2.76 & 1.3 & 0.95 & 135 & 43 & 11.82 \\
\hline UGC 3804 & 13.17 & 0.64 & 1.93 & 12.76 & 0.50 & 1.63 & 1.14 & 0.72 & 13 & 51 & - \\
\hline NGC 2532 & 12.95 & 0.53 & 1.91 & 12.65 & 0.44 & 1.71 & 2.8 & 2.6 & 130 & 22 & (2) \\
\hline NGC 2712 & 12.79 & 0.65 & 2.06 & 12.39 & 0.54 & 1.80 & 3.2 & 1.5 & 0 & 62 & 11.82 \\
\hline IC 529 & 12.50 & 0.48 & 1.56 & 11.95 & 0.35 & 1.21 & 3.3 & 1.45 & 140 & 64 & - \\
\hline NGC 3055 & 12.66 & 0.53 & 1.78 & 12.24 & 0.42 & 1.50 & 2.17 & 1.3 & 63 & 53 & - \\
\hline NGC 3549 & 12.88 & 0.70 & 2.03 & 12.20 & 0.53 & 1.58 & 2.79 & 0.97 & 27 & 70 & - \\
\hline NGC 3811 & 12.80 & 0.51 & 1.77 & 12.59 & 0.45 & 1.63 & 2.79 & 2.07 & 160 & 42 & - \\
\hline NGC 3835 & 13.25 & 0.73 & 2.15 & 12.73 & 0.59 & 1.81 & 2.43 & 0.93 & 56 & 67 & - \\
\hline NGC 5962 & 11.92 & 0.68 & 2.00 & 11.58 & 0.59 & 1.78 & 3.1 & 2.27 & 105 & 43 & - \\
\hline NGC 6155 & 13.07 & 0.49 & 1.48 & 12.82 & 0.41 & 1.32 & 1.65 & 1.1 & 140 & 48 & 12.05 \\
\hline NGC 6395 & $11.98^{*}$ & - & - & $11.65^{*}$ & - & - & 2.59 & 0.93 & 20 & 69 & 12.13 \\
\hline NGC 6643 & 11.77 & 0.54 & 1.73 & 11.07 & 0.37 & 1.29 & 3.62 & 1.96 & 50 & 57 & 11.31 \\
\hline NGC 2341 & 14.01 & 0.61 & 2.26 & 13.50 & 0.46 & 1.95 & 1.0 & 0.87 & 55 & 35 & (2) \\
\hline NGC 2342 & 13.01 & 0.63 & 1.99 & 12.75 & 0.54 & 1.81 & 1.24 & 0.93 & 40 & 41 & (2) \\
\hline NGC 2648 & 12.74 & 0.88 & 2.59 & 12.11 & 0.71 & 2.20 & 3.2 & 1.2 & 145 & 68 & - \\
\hline A $0840+1427$ & 14.33 & 0.35 & 1.05 & 13.17 & 0.06 & 0.32 & 0.83 & 0.21 & 103 & 75 & - \\
\hline NGC 3646 & 11.82 & 0.42 & - & 11.44 & 0.31 & - & 3.8 & 2.17 & 50 & 55 & (3) \\
\hline NGC 3649 & 14.18 & - & 2.46 & 13.79 & - & 2.21 & 1.45 & 0.57 & 140 & 67 & - \\
\hline $\begin{array}{l}\text { NGC } 3690^{* *} \\
\text { IC } 694\end{array}$ & 12.26 & 0.49 & 2.09 & 12.25 & 0.47 & 2.10 & - & - & - & - & 10.88 \\
\hline NGC 5303a & 13.13 & 0.37 & 1.26 & 12.89 & 0.31 & 1.10 & 1.14 & 0.72 & 83 & 51 & 12.01 \\
\hline NGC 5303b & 14.96 & 0.12 & 1.04 & 14.24 & -0.06 & 0.58 & 0.88 & 0.47 & 110 & 58 & 13.04 \\
\hline NGC 7469 & 13.00 & 0.47 & 0.77 & 12.69 & 0.36 & 0.55 & 1.6 & 1.07 & 127 & 48 & 11.44 \\
\hline IC 5283 & 14.67 & 0.61 & 2.04 & 14.31 & 0.50 & 1.79 & 0.72 & 0.36 & 120 & 60 & 12.4 \\
\hline NGC 7537 & 13.70 & 0.59 & 2.01 & 12.61 & 0.31 & 1.33 & 2.06 & 0.57 & 70 & 74 & 12.53 \\
\hline NGC 7541 & 12.37 & 0.66 & 2.12 & 11.46 & 0.45 & 1.55 & 3.1 & 0.98 & 95 & 72 & 11.99 \\
\hline
\end{tabular}

(*) I magnitude.

(**) For the whole system. No correction for internal extinction.

(1) We detect just [NII] emission.

(2) Only line+continuum image available.

(3) Only continuum image available.

which the differences between both branches of the rotation curve, in particular for the most symmetric region which normally corresponds to the central parts, are minimized. Generally, the kinematic center corresponds, within our resolution, to the photometric center, that is to say, the most luminous section in the spectral continuum. Nevertheless, for NGC 6155 the difference amounts to $\approx 3^{\prime \prime}$; this result can be explained as due to the presence of dust, associated with the spiral arms (the spiral structure in this galaxy seem to reach the very central parts).

The redshift corresponding to the kinematical center has been adopted as the redshift of the system in all cases. To calculate the distances we have used the velocities corrected for galactocentric motion as in the $\mathrm{RC} 2$ catalogue $(\Delta V=300 \sin (l) \cos (b))$. We notice that since the galaxies in both samples are nearby ones, the contributions to the observed redshift due to the galactic proper motions could be important. To calculate the distances we have proceeded as follows. We have calculated the distances both considering the effect of a Virgo inflow (model 1 in Kraan-Korteweg 1986) and directly from the observed redshift. The difference between both values is less than $\approx 15 \%$ (excepting for NGC 4525, with $38 \%$ ). Both determinations are comparable from the point of view of the model accuracy and are consequently equivalent for our purposes. We have therefore decided to use the value directly derived from the redshift as a good estimation for the distance; we give this parameter in Table 10.

The rotation curves are described by a number of parameters. They are given in Table 10 and shown in Figs. 2 (for the galaxies with photometric data) and Fig. 4 (for those with no images). The data for those galaxies without photometric information are also included. We have determined the distance to the center, $R_{\max }$, for which the observed velocity reaches a first local maximum, $V_{\max }$. These parameters are given, as observed, in Table 10. Another relevant parameter is the slope, $\mathrm{G}$ (in $\mathrm{km} \mathrm{s}^{-1} / \mathrm{Kpc}$ ), of the inner linear part of the rotation curve, (i.e., the region of rigid solid body rotation, inclination corrected). 
Table 8. Parameters of the bulge/disk decomposition

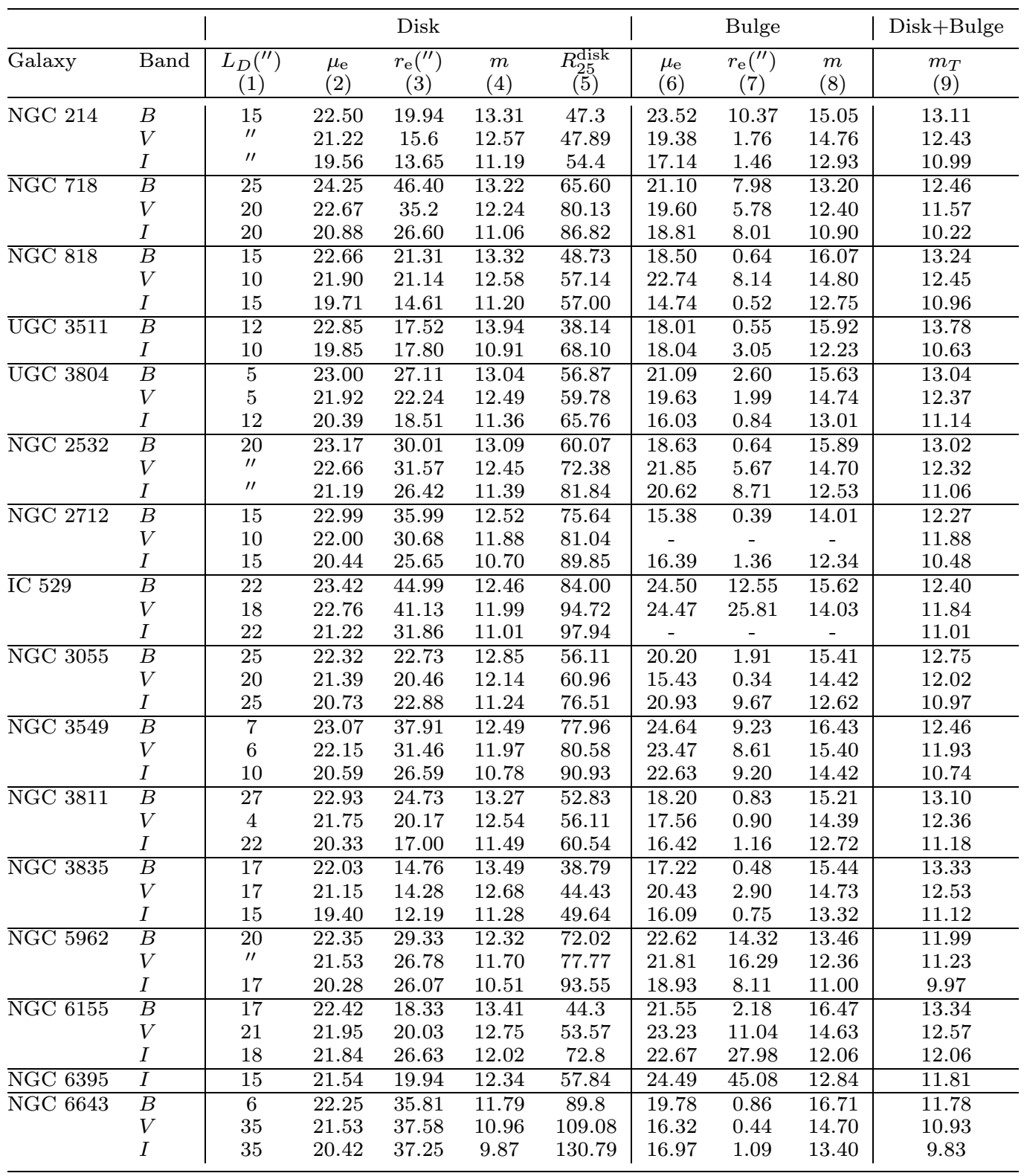

(1) The "marking-the-disk" region (from $L_{D}$ outwards).

(2) Effective surface brightness for the disk component.

(3) Effective radius for the disk component.

(4) Total magnitude for the disk.

(5) Isophotal radius for the disk component at $25 \mathrm{mag} /\left({ }^{\prime \prime}\right) 2$.

(6) Same as (2), for the bulge component.

(7) Same as (3), for the bulge component.

(8) Same as (4), for the bulge component.

(9) Total magnitude as given by the addition of bulge and disk components. 
Table 9. The same as Table 8 for pairs

\begin{tabular}{|c|c|c|c|c|c|c|c|c|c|c|}
\hline \multirow[b]{2}{*}{$\overline{\text { Galaxy }}$} & \multirow[b]{2}{*}{ Band } & \multicolumn{5}{|c|}{ Disk } & \multicolumn{3}{|c|}{ Bulge } & \multirow{2}{*}{$\begin{array}{c}\text { Disk }+ \text { Bulge } \\
m_{T} \\
(9)\end{array}$} \\
\hline & & $\begin{array}{c}L_{D}\left({ }^{\prime \prime}\right) \\
(1)\end{array}$ & $\begin{array}{l}\mu_{\mathrm{e}} \\
(2)\end{array}$ & $\begin{array}{c}r_{\mathrm{e}}\left({ }^{\prime \prime}\right) \\
(3)\end{array}$ & $\begin{array}{c}m \\
(4)\end{array}$ & $\begin{array}{c}R_{25}^{\text {disk }} \\
(5)\end{array}$ & $\begin{array}{l}\mu_{\mathrm{e}} \\
(6)\end{array}$ & $\begin{array}{c}r_{\mathrm{e}}\left({ }^{\prime \prime}\right) \\
(7)\end{array}$ & $\begin{array}{c}m \\
(8)\end{array}$ & \\
\hline \multirow[t]{3}{*}{ NGC 2341} & $B$ & - & - & - & - & - & - & - & - & - \\
\hline & V & 5 & 20.017 & 5.45 & 13.64 & 20.35 & - & - & - & 13.64 \\
\hline & $I$ & 5 & 18.65 & 5.64 & 12.20 & 25.3 & 17.18 & 1.05 & 13.68 & 11.95 \\
\hline \multirow{3}{*}{ NGC 2342} & $B$ & 2 & 22.33 & 20.96 & 13.03 & 51.6 & 15.43 & 0.17 & 15.87 & 12.96 \\
\hline & $V$ & 5 & 21.50 & 18.79 & 12.44 & 54.86 & 16.63 & 0.33 & 15.67 & 12.38 \\
\hline & $I$ & 7 & 20.07 & 16.73 & 11.26 & 62.0 & 16.04 & 0.68 & 13.49 & 11.13 \\
\hline \multirow[t]{3}{*}{ NGC 2648} & $B$ & 15 & 23.83 & 22.30 & 14.39 & 36.7 & 21.38 & 9.63 & 13.07 & 12.78 \\
\hline & $V$ & 20 & 23.62 & 23.60 & 14.06 & 41.53 & 20.40 & 10.13 & 11.99 & 11.84 \\
\hline & $I$ & " & 21.71 & 34.54 & 11.32 & 96.9 & 18.30 & 7.92 & 10.42 & 10.03 \\
\hline \multirow[t]{3}{*}{$\overline{\mathrm{A} 0840+1427}$} & $B$ & 3.5 & 22.94 & 9.95 & 15.26 & 21.2 & 15.81 & 0.25 & 15.39 & 14.56 \\
\hline & $V$ & " & 21.89 & 7.89 & 14.71 & 21.35 & 14.74 & 0.17 & 15.20 & 14.18 \\
\hline & $I$ & " & 20.96 & 7.85 & 13.79 & 25.3 & 22.15 & 7.73 & 14.32 & 13.27 \\
\hline \multirow{2}{*}{ NGC 3646} & $B$ & 10 & 23.30 & 69.5 & 11.39 & 134.5 & 18.91 & 1.24 & 15.06 & 11.36 \\
\hline & $V$ & 25 & 22.41 & 50.32 & 11.20 & 121.93 & 18.54 & 1.69 & 14.01 & 11.13 \\
\hline \multirow{2}{*}{ NGC 3649} & $B$ & 10 & 23.45 & 8.47 & 16.11 & 15.68 & 21.45 & 4.97 & 14.58 & 14.34 \\
\hline & $I$ & "I & 20.58 & 6.49 & 13.83 & 22.22 & 18.94 & 4.99 & 12.06 & 11.86 \\
\hline$\overline{\mathrm{NGC}} 7469^{*}$ & $I$ & & 22.56 & 15.47 & 13.92 & 36.18 & - & - & - & 13.92 \\
\hline \multirow[t]{3}{*}{ NGC 7537} & $B$ & 5 & 21.85 & 9.61 & 14.24 & 26.2 & 24.73 & 10.74 & 16.19 & 14.07 \\
\hline & $V$ & " & 21.14 & 8.91 & 13.67 & 27.92 & 24.13 & 19.60 & 14.28 & 13.18 \\
\hline & $I$ & " & 19.71 & 8.28 & 12.42 & 32.3 & 21.76 & 14.91 & 12.50 & 11.71 \\
\hline NGC 7541* & $I$ & 4 & 19.52 & 20.16 & 10.30 & 80.8 & 20.90 & 6.06 & 13.6 & 10.25 \\
\hline
\end{tabular}

(1) to (9) the same as in Table 8

* NGC 5303 a and b show Type II profiles. The same for NGC $7541 B$ and $V$. For NGC 7469 the contribution of the active nucleus avoid a reliable decomposition both in $B$ and $V$ bands.

This parameter could be connected with the morphological type, being greater for early spirals (Baiesi-Pillastrini 1987). We also give the rotational velocities, $V_{\mathrm{M}}$, for the last measured point, $R_{\mathrm{M}}$.

We have constructed a parameter to give a global description of the shape of the rotation curve. This is defined as $\Delta=\operatorname{tg}^{-1}\left[\left(V_{\mathrm{M}}-V_{\max }\right) /\left(\max \left(V_{\mathrm{M}}, V_{\max }\right)\right) \times\right.$ $\left.\left(R_{\mathrm{M}}-R_{\max }\right) /\left(r_{25}\right)\right]$, i.e. the velocities are normalised to the maximum amplitude and the radii are normalised to $r_{25}$. It can take values around zero (flat rotation curves), positive (for rising rotation curves), or negatives (for declining rotation curves).

The total masses within $R_{\mathrm{M}}$ and $r_{25}$ (this last from the RC3 catalogue), for an homogenous and spherically symmetric distribution $\left(M_{\mathrm{T}}=2.326510^{5} r v^{2}(r) M_{\odot}\right.$, with $r$ in $\mathrm{Kpc}$ and $v(r)$ in $\mathrm{km} \mathrm{s}^{-1}$ ) are also given. Inclination corrections were applied following Peterson et al. (1978).

For some cases the minor axis spectrum is also available (see Tables 1 and 2). The resulting velocity distributions are plotted in Fig. 5. When non-circular motions are present, they are obviously more easily detected through the spectra along the minor axis. Therefore, this kind of data is specially interesting to detect the presence of structures that can easily produce them, as bars or rings. This information will be used together with the masking, the simulated images and the Fourier analysis in order to give a more precise morphological classification.

For some galaxies the worked out photometric decomposition could be used to produce a good fit to the ob- served rotation curve. Those cases are presented in Fig. $2 \mathrm{~h}$ where the individual $M / L$ ratios for bulge and disk components are given. They are calculated under the hypothesis of maximum disk, that is to say, assuming that the maximum rotation velocity is due to the disk contribution. Those fits and the other unfitted cases will be discussed in the forthcoming papers.

\subsubsection{Line ratios}

We already said that the spectroscopic data were not flux calibrated. However, due to the proximity in wavelength between the [NII], [SII] and $\mathrm{H} \alpha$ lines, it seems possible to neglect the extinction and calibration differential effects and directly compare the fluxes of these lines. In that way, a crude estimation of the physical state of the ionized gas can be made. The line ratios are collected in Tables 11 and 12. The analysis of those data will be presented in Paper III.

We report here on three objects that could show some kind of low level nuclear activity. None of them is in the Véron-Cetty \& Véron Catalogue (1993). In the nuclear region of NGC 718 there is a strong absorption under $\mathrm{H} \alpha$. The $\mathrm{H} \alpha$ emission is weak and the $[\mathrm{NII}] \lambda 6583 / \mathrm{H} \alpha$ ratio is high. The possibility for it being an active nuclei is reinforced by the fact that the observed [SII] line fluxes are indicative of rather high density $([\mathrm{SII}] \lambda 6717 /[\mathrm{SII}] \lambda 6731=$ 

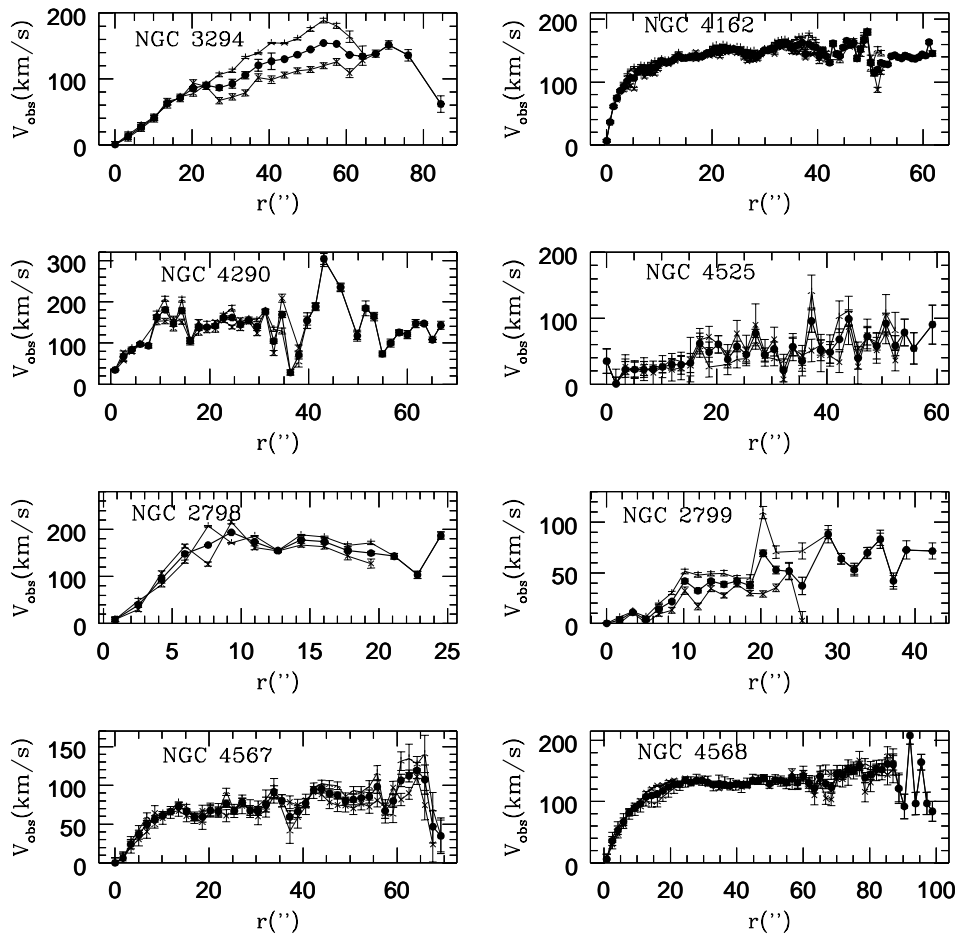

Fig. 4. Rotation curves (average of both sides) for the galaxies with no images. Isolated spirals: NGC 3294, NGC 4162, NGC 4290 and NGC 4525. Interacting galaxies: the pairs NGC 2798/99 and NGC 4567/68

0.73). The $[\mathrm{OI}] \lambda 6300$ line is also detected. Our results are consistent with the spectrum shown in Ho et al. (1995).

NGC 3835 also has a very high $[\mathrm{NII}] \lambda 6583 / \mathrm{H} \alpha$ ratio. In that galaxy the absorption under $\mathrm{H} \alpha$ is not as prominent as in the previous object, even if it is present. The point here is that $[\mathrm{NII}] \lambda 6583$ is extended and dominates over $\mathrm{H} \alpha$ along the 4 central arcseconds. The $[\mathrm{OI}] \lambda 6300$ line is detected and $[\mathrm{SII}] \lambda 6717 /[\mathrm{SII}] \lambda 6731$ is $\approx 0.95$, indicating a moderately high electronic density. Thus, NGC 3835 could also harbor an active nucleus.

Finally, the line ratios we find for NGC 5962 are also indicative of some kind of nuclear activity. In the central region we find $[\mathrm{NII}] \lambda 6583 / \mathrm{H} \alpha$ over 1 . However the Balmer absorptions are very important (see also Ho et al.) and a detailed analysis has to be done before concluding on the nature of the nucleus of that galaxy. The $[\mathrm{SII}] \lambda 6717 /[\mathrm{SII}] \lambda 6731$ lines ratio is $\approx 0.95$. No $[\mathrm{OI}]$ lines are detected.

\section{Comparison with RC3 values}

In Fig. 6 we plot the difference between the apparent $B$ magnitude we have measured, $m_{B}$ (data), versus that given in the $\mathrm{RC} 3, m_{B}(\mathrm{RC} 3)$, for isolated galaxies (full triangles) and spirals in pairs (open squares). The displayed error bars are those given in the RC3 catalogue. It can be seen that significant discrepancies are found only for an isolated galaxy, UGC 3511, and four interacting objects, NGC 3649, NGC 5303b, IC 5283 and A 0840+1417 (not plotted, see below).

It is not possible to make an internal control of the quality of our data for the two first galaxies, UGC 3511 and NGC 3649, since their $V$ images are not available. We note however that the $(B-I)$ color we find for NGC 3649 corresponds rather well to what would be expected for its morphological type (SBS1). On the other hand, part of the discrepancy could result from the different ways the bright star projected on the galaxy has been eliminated. For UGC 3511 the situation is more uncertain, since the $(B-I)$ value we find for it is redder than what is normally found for Scd galaxies.

For NGC 5303b, the data for the stars in the same frames indicate that our photometry is accurate within $2 \sigma$ of the standard star values. This makes us confident on the quality of our data. Moreover, the difference between our value and the RC3 one for NGC 5303a, observed in the same frame as its companion, is well within the errors reported in the RC3 for that galaxy.

The case of IC 5283 was already discussed in Márquez $\&$ Moles (1994), in the sense of being preferable the values we report here. Finally, for A $0840+1417$ the difference amounts to $-1.07 \mathrm{mag}$ (out of the plot scale). We can 

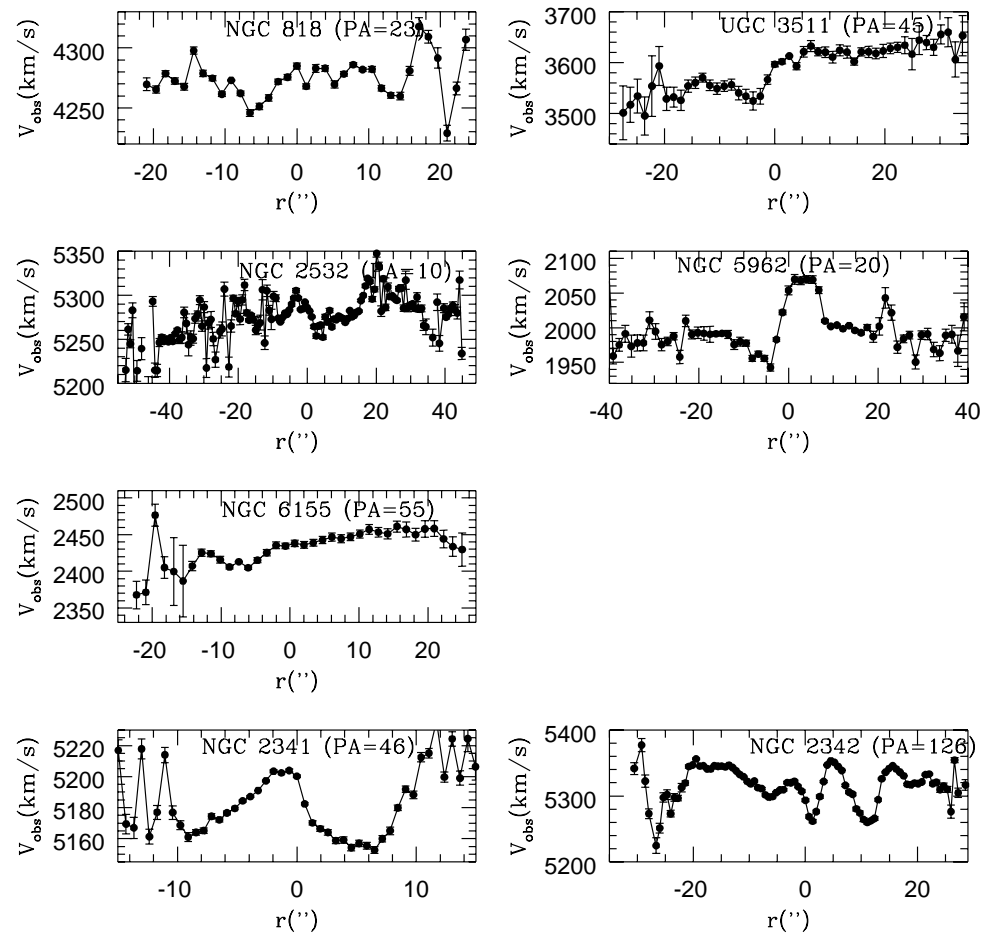

Fig. 5. Velocity distribution along the minor axis. Isolated spirals: NGC 818, UGC 3511, NGC 2532 and NGC 6155. Interacting galaxies: NGC 2341 and NGC 2342

argue, first, that the stars in the corresponding frames give $\sigma_{B V / V I}^{\text {foreg }}=0.05$ and $\sigma_{B V / V I}^{\text {foreg }}=0.03$, so we are very confident on the calibration. Secondly, for NGC 2648, observed in the same frame as A $0840+1417$, the agreement between our data and those reported in the RC3 is very good.

All in all, and with the possible exception of UGC 3511 for which we do not have enough controls, we think that our photometry is accurate at better than 0.1 mag. Indeed, leaving aside these 4 highly discrepant cases, the rms of the differences between our data and the RC3 values amount to $0.09 \mathrm{mag}$ for the sample of isolated galaxies, and to 0.19 mag for the interacting galaxies. The individual differences are always smaller than the errors reported in the RC3, excepting for NGC 7541, for which it is about twice the reported error $(0.1 \mathrm{mag})$. We note that the $\mathrm{rms}$ of the photometry of the stars in the corresponding frames is low (about $0.04 \mathrm{mag}$ ). Moreover, the difference for NGC 7537 , observed in the same frame, amounts to only 0.05 mag, what makes us confident on the accuracy of our data for NGC 7541.

Taking both samples together, the rms of the differences amounts to 0.14 mag, a well acceptable value given the uncertainties in both sets of data.

In Fig. 7 we make a similar comparison for the color index $(B-V)$. The galaxy showing the highest difference is IC 5283. This galaxy is a companion to NGC 7469 and was observed together with the main galaxy, in the same frame actually. The data for NGC 7469 are in very good agreement with those reported in the RC3 catalogue, what give us confidence on the accuracy of the data for IC 5283. On the other hand, the colors we find are much more reasonable for a late type galaxy as IC 5283 (Márquez \& Moles 1994).

The comparison of the position angles given by the catalogue and determined by our data is given in Fig. 8 . The differences are always smaller than $10^{\circ}$, excepting for NGC 2532, NGC 2341 and NGC 2342, for which the discrepancy is rather high, and A0840+1427 with PA(data)$\mathrm{PA}(\mathrm{RC} 3)=24^{\circ}$. For the last object, the difference could be simply due to the difficulties in its PA determination caused by the disk warping. For 2532, NGC 2341 and NGC 2342 we can argue that both photometric and kinematical PA, as determined here, are in very good agreement, what makes us confident in our results. We therefore conclude that our data are accurate within a few degrees.

To calculate the inclinations we have considered intrinsically circular disks. In Fig. 9 we see that there is a general agreement between our values and those reported in the RC3 (the error bars are those quoted in the RC3 catalogue). The differences are always smaller than $10^{\circ}$, excepting NGC 2341 and NGC 2342, just discussed above (see also notes on individual galaxies). 
Table 10. Parameters from the rotation curves

\begin{tabular}{|c|c|c|c|c|c|c|c|c|c|c|c|c|c|}
\hline Galaxy & $\begin{array}{c}c z \\
(1)\end{array}$ & $\begin{array}{r}D \\
(2)\end{array}$ & $\begin{array}{c}r_{G} \\
(3)\end{array}$ & $\begin{array}{c}v_{G}^{\mathrm{ob}} \\
(4)\end{array}$ & $\begin{array}{l}\mathrm{G} \\
(5)\end{array}$ & $\begin{array}{r}r_{\max } \\
(6)\end{array}$ & $v_{\max }^{\mathrm{ob}}$ & $\begin{array}{c}r_{\mathrm{M}} \\
(8)\end{array}$ & $\begin{array}{r}r_{\mathrm{M}} / r_{25} \\
(9)\end{array}$ & $\begin{array}{c}v_{\mathrm{M}}^{\mathrm{ob}} \\
(10)\end{array}$ & $\begin{array}{c}\Delta \\
(11)\end{array}$ & $\begin{array}{l}M_{\mathrm{M}} \\
(12)\end{array}$ & $\begin{array}{l}M_{T} \\
(13)\end{array}$ \\
\hline NGC 718 & 1720 & 24 & - & - & - & - & - & & & & - & & \\
\hline NGC 818 & 4273 & 55.5 & 4 & 130 & 132 & 20 & 219 & 49 & 0.55 & 220 & 0 & 17.8 & 32.3 \\
\hline UGC 3511 & 3583 & 49.6 & 2.9 & 73 & 158 & 12 & 120 & 33 & 0.74 & 122 & 2 & 5.9 & 8.0 \\
\hline UGC 3804 & 2922 & 41.8 & 3.6 & 115 & 208 & 12 & 139 & 49 & 0.92 & 142 & 2 & 7.7 & 8.4 \\
\hline NGC $2532^{*}$ & 5274 & 70.5 & 2.3 & 29 & 125 & 20 & 75 & 39 & 0.59 & 82 & 17 & 21 & 35.0 \\
\hline NGC 2712 & 1835 & 25.7 & 2.5 & 106 & 385 & 20 & 150 & 49 & 0.57 & 152 & 2 & 4.2 & 7.4 \\
\hline IC 529 & 2296 & 33.4 & 4.2 & 46 & 75 & 40 & 120 & 80 & 0.73 & 140 & 21 & 7.3 & 10.0 \\
\hline NGC 3055 & 1873 & 23.4 & 3.5 & 27 & 85 & 40 & 110 & 50 & 0.80 & 116 & 18 & 2.8 & 3.5 \\
\hline NGC 3549 & 2863 & 39.9 & 8.9 & 80 & 50 & 20 & 180 & 80 & 0.82 & 210 & 13 & 20.3 & 24.5 \\
\hline NGC 3811 & 3081 & 42.5 & 1.4 & 56 & 290 & 20 & 120 & 62 & 0.94 & 122 & 1 & 9.9 & 10.5 \\
\hline NGC 3835 & 2453 & 34.9 & 0.9 & 32 & 227 & 25 & 190 & 48 & 0.82 & 198 & 6 & 8.7 & 10.6 \\
\hline NGC 5962 & 1983 & 27.6 & 3.4 & 144 & 464 & 20 & 165 & 63 & 0.71 & 167 & 1 & 11.8 & 16.6 \\
\hline NGC 6155 & 2433 & 35.3 & 1.5 & 30 & 157 & 12 & 80 & 41 & 1.00 & 90 & 9 & 2.4 & 2.4 \\
\hline NGC 6395 & 1130 & 18.6 & 4.1 & 14 & 41 & 60 & 110 & 78 & 1.08 & 115 & 10 & 2.5 & 2.3 \\
\hline NGC 6643 & 1463 & 23.0 & 2.7 & 50 & 202 & 40 & 160 & 94 & 0.82 & 164 & 3 & 11.3 & 13.8 \\
\hline NGC 3294 & 1567 & 20.7 & 10 & 41 & 48 & 54 & 152 & 68 & 0.6 & 150 & -5 & 4.9 & 7.6 \\
\hline NGC 4162 & 2557 & 33.6 & 1.3 & 61 & 360 & 22 & 159 & 48 & 0.69 & 160 & 0 & 7.3 & 10.6 \\
\hline NGC 4290 & 3053 & 42.3 & 0.8 & 35 & 297 & 11 & 180 & 31 & 0.44 & 180 & 0 & 9.3 & 21.0 \\
\hline NGC 4525 & 1185 & 15.8 & - & - & - & 20 & 65 & 50 & 0.65 & 70 & 10 & 0.6 & 0.9 \\
\hline NGC $2341^{* *}$ & 5192 & 70.3 & 0.96 & 57 & 308 & 10 & 143 & 20 & 0.80 & 90 & -41 & 10.2 & 12.8 \\
\hline NGC 2342 & 5314 & 70.3 & 1.3 & 89 & 307 & 9 & 150 & 35 & 0.85 & 152 & 1 & 15.1 & 17.7 \\
\hline NGC $2648^{* *}$ & 2052 & 27.4 & 2.26 & 154 & 553 & 10 & 200 & 30 & 0.3 & 125 & -61 & 4.3 & 14.3 \\
\hline A $0840^{*}$ & 2160 & 27.4 & 3 & 7 & 20 & 10 & 30 & 15 & 0.57 & 2 & -78 & 0.3 & 0.6 \\
\hline NGC 3646 & 4242 & 55.8 & 2.14 & 162 & 342 & 70 & 260 & 100 & 0.86 & 260 & 0 & 63.4 & 73.7 \\
\hline NGC 3690 & 3017 & 42.9 & & $\cdot$ & & 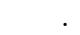 & & & 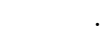 & 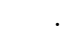 & 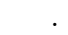 & . & \\
\hline IC 694 & 3117 & 42.9 & & & & & & & & & & & \\
\hline NGC 5303a & 1447 & 20.6 & 2.7 & 41 & 196 & 10 & 80 & 20 & 0.73 & 96 & 25 & 0.71 & 0.97 \\
\hline NGC 5303b & 1421 & 20.6 & 1.35 & 11 & 96 & 13 & 55 & 20 & 0.92 & 46 & -27 & 0.19 & 0.2 \\
\hline NGC $7469^{* *}$ & 4898 & 68 & 5.5 & 121 & 92 & 5.5 & 121 & 15 & 0.22 & 72.5 & -63 & 3.3 & 15.0 \\
\hline IC 5283 & 4877 & 68 & 3 & 51 & 60 & 8.8 & 151 & 20 & 0.84 & 155 & 3 & 4.9 & 5.8 \\
\hline NGC 7537 & 2642 & 38 & 7.5 & 104 & 79 & 17 & 137 & 65 & 0.97 & 145 & 4 & 9.6 & 9.9 \\
\hline NGC 7541 & 2653 & 38 & 2 & 119 & 342 & 50 & 198 & 87 & 0.84 & 200 & 2 & 20.3 & 24.2 \\
\hline NGC $2798^{* *}$ & 1789 & 24.5 & 2.5 & 39 & 142 & 9 & 194 & 19 & 0.25 & 150 & -60 & 2.3 & 9.2 \\
\hline NGC 2799 & 1880 & 24.5 & 3.4 & 11 & 28 & 10 & 41 & 22 & 0.39 & 50 & 40 & 0.2 & 0.4 \\
\hline NGC $4567^{* *}$ & 2282 & 29.2 & 6.7 & 50 & 72 & 64 & 119 & 67 & 0.74 & 47 & -85 & 5.8 & 7.9 \\
\hline NGC 4568 & 2251 & 29.2 & 2.6 & 36 & 109 & 24 & 135 & 87 & 0.64 & 160 & 19 & 9.1 & 14.2 \\
\hline
\end{tabular}

* Corrected to the galactic plane

** Declining curve. Mass determined through $V_{\max }$

(1) Observed systemic velocity $(\mathrm{km} / \mathrm{s})$.

(2) Distance with $H_{0}=75 \mathrm{kms}^{-1} \mathrm{Mpc}^{-1}$ and $\Delta V=300 \sin (l) \cos (b)$.

(3) Radius for inner region of solid-body rotation ( $\left.{ }^{\prime \prime}\right)$.

(4) Observed velocity amplitud for $r_{G}(\mathrm{~km} / \mathrm{s})$.

(5) $G=\left(v_{G}^{\mathrm{ob}} / \sin (i)\right) / r_{G}(\mathrm{Kpc})\left(\mathrm{km} \mathrm{s}^{-1} \mathrm{kpc}^{-1}\right)$.

(6) Radius for the maximum rotation velocity, $v_{\max }^{\mathrm{ob}}\left({ }^{\prime \prime}\right)$.

(7) Observed maximum rotation velocity $(\mathrm{km} / \mathrm{s})$.

(8) Last measured point in the rotation curve (").

(9) $r_{\mathrm{M}} / r_{25}$, with $r_{25}$ as given in the RC3.

(10) Last measured observed velocity in the rotation curve $(\mathrm{km} / \mathrm{s})$.

(11) Shape of the rotation curve (see text).

(12) Mass inside $r_{\mathrm{M}}\left(10^{10} M_{\odot}\right)$.

(13) Mass inside $r_{25}\left(10^{10} M_{\odot}\right)$.

In Fig. 10 we plot the differences between the redshifts determined by us and those given by other optical determinations as reported in RC3. Isolated galaxies are represented by full triangles and interacting ones by open squares; open triangles correspond to isolated galaxies with only $21 \mathrm{~cm}$ redshift data reported in the RC3.
For all of them we have plotted only the error bars for the RC3 data.

Among the isolated galaxies, the agreement is generally good excepting 4 galaxies, namely IC $529(\Delta v=205$ $\left.\mathrm{km} \mathrm{s}^{-1}\right)$, NGC $818\left(\Delta v=-184 \mathrm{~km} \mathrm{~s}^{-1}\right)$, NGC $2532(\Delta v=$ $\left.121 \mathrm{~km} \mathrm{~s}^{-1}\right)$ and NGC $3294\left(\Delta v=131 \mathrm{~km} \mathrm{~s}^{-1}\right)$. Otherwise the differences are small (average $=20.5 \mathrm{~km} \mathrm{~s}^{-1}$ ), with a 
Table 11. Line parameters (isolated galaxies)

\begin{tabular}{|c|c|c|c|c|c|c|c|c|c|c|c|}
\hline Galaxy & $\mathrm{EW}(\mathrm{H} \alpha)$ & {$[\mathrm{NII}] / \mathrm{H} \alpha$} & Reg. & | Galaxy & $\mathrm{EW}(\mathrm{H} \alpha)$ & {$[\mathrm{NII}] / \mathrm{H} \alpha$} & Reg. & | Galaxy & $\mathrm{EW}(\mathrm{H} \alpha)$ & {$[\mathrm{NII}] / \mathrm{H} \alpha$} & Reg. \\
\hline \multirow[t]{4}{*}{ N818 } & 10. & 0.47 & ext. & \multirow[t]{3}{*}{ N3811 } & 10. & 0.56 & ext. & \multirow[t]{6}{*}{ N3294 } & 24.2 & 0.38 & ext. \\
\hline & 11. & 0.38 & ext. & & 10. & 0.53 & nuc. & & 27.8 & 0.40 & ext. \\
\hline & 15. & 0.40 & ext. & & 16. & 0.48 & ext. & & 22.7 & 0.45 & nuc. \\
\hline & 38. & 0.42 & ext. & \multirow[t]{7}{*}{ N3835 } & 4. & 0.32 & ext. & & 32.4 & 0.42 & nuc. \\
\hline \multirow[t]{4}{*}{ U3511 } & 54. & 0.37 & ext. & & 13. & 0.45 & ext. & & 24.9 & 0.45 & ext. \\
\hline & 43. & 0.33 & ext. & & 8. & 0.55 & ext. & & 42.5 & 0.44 & ext. \\
\hline & 10. & 0.55 & nuc. & & 2. & 2.78 & nuc. & N4162 & 69.4 & 0.69 & ext. \\
\hline & 17. & 0.49 & ext. & & 6. & 0.60 & ext. & & 29.7 & 0.60 & ext. \\
\hline \multirow[t]{6}{*}{ U3804 } & 17. & 0.41 & ext. & & 13. & 0.46 & ext. & & 22.7 & 0.48 & ext. \\
\hline & 16. & 0.39 & ext. & & 7. & 0.54 & ext. & & 23.1 & 0.39 & ext. \\
\hline & 8. & 0.50 & ext. & \multirow[t]{6}{*}{ N5962 } & 20. & 0.46 & ext. & & 13.8 & 0.34 & ext. \\
\hline & 12. & 0.42 & ext. & & 45. & 0.33 & ext. & & 3.6 & 0.15 & nuc. \\
\hline & 9. & 0.47 & ext. & & 13. & 0.53 & ext. & & 8.9 & 0.40 & ext. \\
\hline & 33. & 0.30 & ext. & & 4. & 0.76 & nuc. & & 20.7 & 0.41 & ext. \\
\hline \multirow[t]{4}{*}{ N2532 } & 111. & 0.26 & ext. & & 15. & 0.42 & ext. & & 53.7 & 0.49 & ext. \\
\hline & 22 & 0.33 & ext. & & 41. & 0.38 & ext. & N4290 & 20.4 & 0.27 & nuc. \\
\hline & 18. & 0.41 & nuc. & \multirow[t]{5}{*}{ N6155 } & 15. & 0.41 & ext. & $\mathrm{N} 4525$ & 5.0 & 0.41 & nuc. \\
\hline & 22 . & 0.32 & ext. & & 11. & 0.47 & nuc. & & 9.0 & 0.75 & ext. \\
\hline \multirow{6}{*}{$\mathrm{N} 2712$} & 8. & 0.34 & ext. & & 32. & 0.35 & nuc. & & & & \\
\hline & 7. & 0.49 & ext. & & 13. & 2.89 & ext. & & & & \\
\hline & 5. & 0.55 & ext. & & 13. & 0.40 & ext. & & & & \\
\hline & 19. & 0.48 & nuc. & \multirow[t]{3}{*}{ N6395 } & 17. & 0.13 & ext. & & & & \\
\hline & 12. & 0.43 & ext. & & 38. & 0.16 & ext. & & & & \\
\hline & 55. & 0.40 & ext. & & 48. & 0.15 & nuc. & & & & \\
\hline \multirow[t]{4}{*}{ I529 } & 13. & 0.47 & ext. & & 20. & 0.21 & ext. & & & & \\
\hline & 10. & 0.41 & nuc. & \multirow{10}{*}{ N6643 } & 50. & 0.19 & ext. & & & & \\
\hline & 17. & 0.23 & ext. & & 11. & 0.41 & ext. & & & & \\
\hline & 19. & 0.31 & ext. & & 14. & 0.43 & ext. & & & & \\
\hline N3055 & 11. & 0.43 & nuc. & & 19. & 0.37 & ext. & & & & \\
\hline \multirow[t]{6}{*}{ N3549 } & 9. & 0.40 & ext. & & 13. & 0.40 & ext. & & & & \\
\hline & 8. & 0.39 & ext. & & 38. & 0.31 & ext. & & & & \\
\hline & 8. & 0.45 & ext. & & 14. & 0.69 & nuc. & & & & \\
\hline & 1. & 2.45 & nuc. & & 23. & 0.38 & ext. & & & & \\
\hline & 6. & 0.49 & ext. & & 10. & 0.39 & ext. & & & & \\
\hline & 16. & 0.38 & ext. & & 74 . & 0.31 & ext. & & & & \\
\hline
\end{tabular}

ext. $=$ external region

nuc. $=$ nuclear region

rms value of $19.7 \mathrm{kms}^{-1}$. Since the galaxies are isolated it seems safe to admit that the optical and HI redshift determinations should be rather concordant. Indeed, direct comparison of those values for the galaxies for which the $\mathrm{RC} 3$ values and ours are in agreement gives for $v_{\mathrm{opt}}-v_{\mathrm{HI}}$ an average of 26.2 , with an rms of $20.9 \mathrm{~km} \mathrm{~s}^{-1}$. This is in agreement with our assumption and let us to use the HI redshift values of the discrepant cases to control the accuracy of the different determinations. Comparing our values and the $\mathrm{HI}$ ones we find much smaller differences than when we compare our results with the optical determinations in the RC3, namely $32 \mathrm{~km} \mathrm{~s}^{-1}, 8 \mathrm{~km} \mathrm{~s}^{-1}, 23$ and $-19 \mathrm{~km} \mathrm{~s}^{-1}$, respectively for the four discrepant galaxies. This adds confidence to the quality of the data we report here.

For the interacting galaxies, the redshift we determined is in good agreement with the (optical) values reported in the RC3 excepting 3 cases: NGC 2648, NGC 2342 and NGC 5303a, with differences of 132,105 and $100 \mathrm{~km} \mathrm{~s}^{-1}$.
For the other interacting galaxies, the average difference is $23.3 \pm 15.6 \mathrm{~km} \mathrm{~s}^{-1}$.

In this case, a comparison with the HI values could be somewhat problematic, in the sense that, for close pairs, some contamination due to the inclusion of part or all the companion galaxy in the beam could be present. Nevertheless, there is just one case for which the difference $v_{\mathrm{opt}}-v_{\mathrm{HI}}$ is as high as $125 \mathrm{kms}^{-1}$ (NGC 2799), what could be due to the inclusion of the companion galaxy, NGC 2798, since $v_{\mathrm{opt}}-v_{\mathrm{RC} 3}^{\mathrm{opt}}=17 \mathrm{~km} \mathrm{~s}^{-1}$. Hence, it seems reasonable to proceed with a comparison with $21 \mathrm{~cm}$ catalogued values. For NGC 2648 the redshift difference is only of $-8 \mathrm{~km} \mathrm{~s}^{-1}$ when our result is compared to the HI redshift value. This coincidence could however somewhat accidental since contamination by the close companion cannot be excluded. A similar result is found for NGC 2342 , with $v_{\mathrm{opt}}-v_{\mathrm{HI}}=38 \mathrm{~km} \mathrm{~s}^{-1}$. For the third discrepant case, that of NGC 5303a, no HI data are available. Leaving aside these 3 galaxies, the differences are $25.4 \mathrm{~km} \mathrm{~s}^{-1}$ in 
Table 12. Line parameters (interacting galaxies)

\begin{tabular}{|c|c|c|c|c|c|c|c|}
\hline Galaxy & $\mathrm{EW}(\mathrm{H} \alpha)$ & {$[\mathrm{NII}] / \mathrm{H} \alpha$} & Reg. \| & Galaxy & $\mathrm{EW}(\mathrm{H} \alpha)$ & {$[\mathrm{NII}] / \mathrm{H} \alpha$} & Reg. \\
\hline N2341 & 37. & 0.39 & nuc. & \multirow[t]{6}{*}{ N7537 } & 43. & 0.29 & ext. \\
\hline \multirow[t]{5}{*}{$\mathrm{N} 2342$} & 138. & 0.28 & ext. & & 50. & 0.35 & ext. \\
\hline & 19. & 0.49 & ext. & & 28. & 0.47 & ext. \\
\hline & 47. & 0.67 & nuc. & & 36. & 0.47 & nuc. \\
\hline & 16. & 0.51 & ext. & & 27. & 0.48 & ext. \\
\hline & 54. & 0.33 & ext. & & 47. & 0.35 & ext. \\
\hline 0840 & 143. & 0.22 & nuc. & \multirow[t]{7}{*}{ N7541 } & 57. & 0.31 & ext. \\
\hline \multirow[t]{6}{*}{ N3646 } & 25. & 0.39 & ext. & & 16. & 0.51 & ext. \\
\hline & 76. & 0.33 & ext. & & 11. & 0.64 & ext. \\
\hline & 7. & 0.56 & ext. & & 15. & 0.58 & nuc. \\
\hline & 7. & 0.42 & ext. & & 26. & 0.42 & ext. \\
\hline & 40. & 0.37 & ext. & & 25. & 0.35 & ext. \\
\hline & 18. & 0.36 & ext. & & 64. & 0.34 & ext. \\
\hline \multirow[t]{3}{*}{ N3690 } & 30. & 0.43 & ext. & N2798 & 76. & 0.28 & nuc. \\
\hline & 75. & 0.38 & ext. & \multirow[t]{3}{*}{ N2799 } & 23. & 0.53 & ext. \\
\hline & 375 . & 0.34 & nuc. & & 50. & 0.56 & nuc. \\
\hline \multirow[t]{3}{*}{ N5303A } & 69. & 0.28 & ext. & & 17. & 0.63 & ext. \\
\hline & 10. & 0.53 & nuc. & \multirow[t]{5}{*}{$\mathrm{N} 4567$} & 18. & 0.45 & ext. \\
\hline & 24. & 0.39 & ext. & & 15. & 0.63 & ext. \\
\hline \multirow[t]{3}{*}{ N5303B } & 33. & 0.19 & ext. & & 6. & 0.32 & nuc. \\
\hline & 37. & 0.13 & nuc. & & 20. & 0.41 & ext. \\
\hline & 82. & 0.10 & ext. & & 7. & 0.32 & ext. \\
\hline N7469 & 96. & 0.67 & nuc. & \multirow[t]{6}{*}{ N4568 } & 16. & 0.48 & ext. \\
\hline & 77. & 0.71 & nuc. & & 22. & 0.49 & ext. \\
\hline \multirow[t]{3}{*}{ I5283 } & 39. & 0.39 & nuc. & & 18. & 0.40 & nuc. \\
\hline & 29. & 0.41 & ext. & & 21. & 0.45 & ext. \\
\hline & 34. & 0.33 & ext. & & 27. & 0.46 & ext. \\
\hline - & - & - & - & & 29. & 0.41 & ext. \\
\hline
\end{tabular}

ext. $=$ external region nuc. $=$ nuclear region

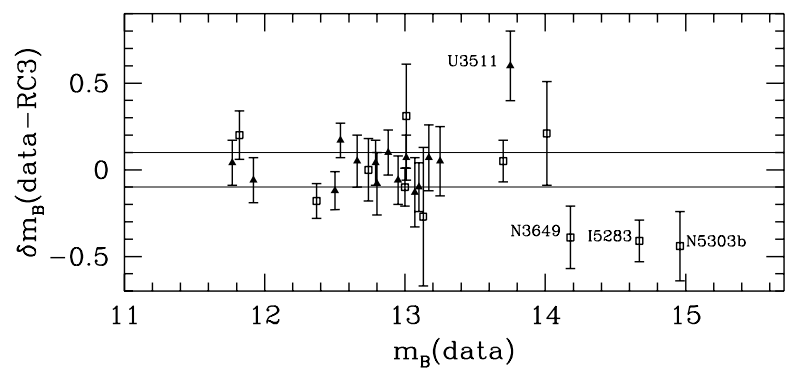

Fig. 6. Comparison between total $B$ magnitudes as derived here $\left(m_{B}\right.$ (data) $)$ with those reported in the RC3 $\left(m_{B}(\mathrm{RC} 3)\right)$. We plot $m_{B}$ (data) versus the difference $\delta m_{B}=m_{B}$ (data) $m_{B}$ (RC3), for isolated galaxies (full triangles) and isolated pairs (open squares). The error bars are those given in the RC3

average with an rms of $19.7 \mathrm{~km} \mathrm{~s}^{-1}$. We therefore conclude that our data for both isolated and interacting galaxies are accurate at the level of $\approx 30 \mathrm{~km} \mathrm{~s}^{-1}$.

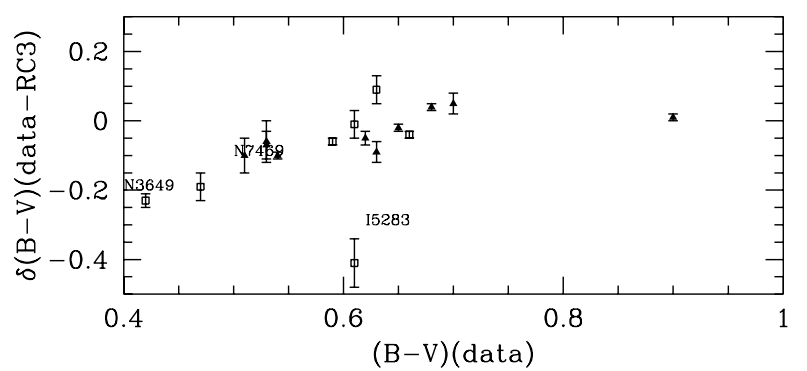

Fig. 7. Same as Fig. 6, for total $(B-V)$ colors

\section{Summary}

In order to analyze the effect of the gravitational interaction in spiral galaxies, we have selected a sample of isolated spirals and a sample of spirals in isolated pairs. Both samples have been selected by using the same restrictions concerning individual galaxies (see Sect. 2). We note that the distribution of morphological subtypes differs considerably for the two samples, a bias that will have to be considered in the comparison.

This paper is devoted to the presentation of the data obtained for the two samples. They include Johnson $B, V$ 


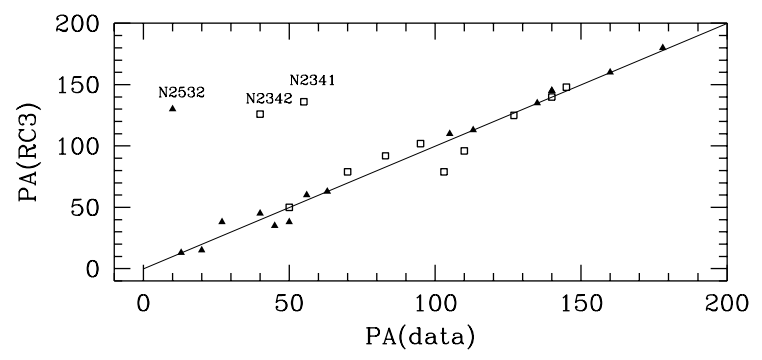

Fig. 8. Plot of our PA determination versus that found in $\mathrm{RC} 3$. The full line represents $\mathrm{PA}(\mathrm{RC} 3)=\mathrm{PA}($ data $)$. See text for labeled galaxies

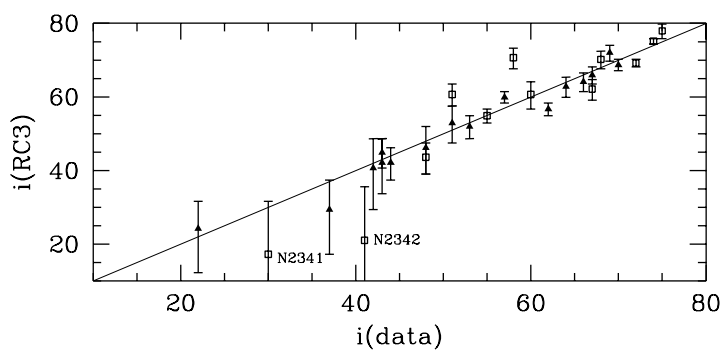

Fig. 9. Same as Fig. 8, for the disk inclination determined as $\cos (i)=b / a$. The full line represents $i(\mathrm{RC} 3)=i($ data $)$. The error bars are those given in the RC3. See text for labeled galaxies

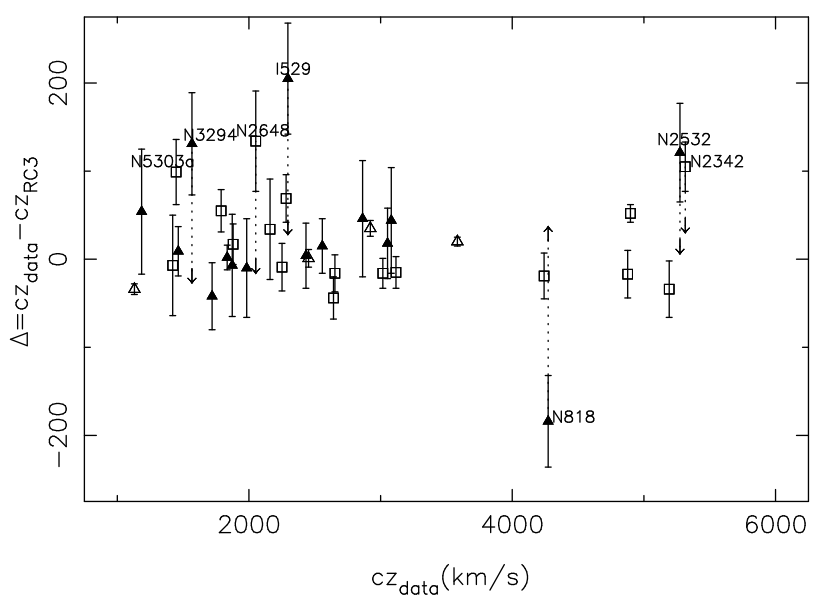

Fig. 10. The difference with RC3 values, $\Delta=c z_{\text {data }}-c z_{\mathrm{RC} 3}$, versus the systemic velocity from our data $\left(c z_{\text {data }}\right)$. Symbols as in Fig. 6. Open triangles correspond to those galaxies for which the only catalogued data in the RC3 are the $21 \mathrm{~cm}$ redshifts. For then cases for which the discrepancy is higher than $100 \mathrm{~km} \mathrm{~s}^{-1}$, we have also plotted the HI data connected by dotted arrows to the corresponding optical values and $I$ images and long slit spectra along the major axes of most of the galaxies, together with $\mathrm{H} \alpha$ images and minor axis spectra for a few of them. We describe the methods we have used for the analysis of the data.

All the isolated galaxies presented here do have structures and features that depart from axial symmetry, in the form of small bars and/or rings. On the other hand, only among interacting galaxies photometric type II profiles and declining rotation curves are found. The detailed analysis of these and other derived properties will be published separately (Papers II and III).

We have shown that the total parameters obtained are generally in good agreement with those given in the RC3 catalogue. Discrepancies have been discussed and the accuracy of our data ascertained. We have completed the morphological characterization of some galaxies and determined completely different values of the position angles of one isolated galaxy (NGC 2532) and two interacting ones (NGC 2341/42) via the combination of photometric and kinematical data (see Appendix A).

We have identified three galaxies, NGC 718, NGC 3835, and NGC 5962, that could present some kind of low level nuclear activity, reported here for the first time.

\section{A. Appendix. Notes on individual galaxies}

Published only in electronic form and available on the server of the Editions de Physique: http://www.ed-phys.fr

Acknowledgements. We gratefully acknowledge Dr. J. Perea, who kindly made the program LINEAS available to us, and Dr. J. Masegosa, who helped us with the analysis of the spectra. We acknowledge financial support by the Spanish DGICYT, under the program PB93-0139. IM, acknowledges a grant from the Spanish Ministerio de Educación y Ciencia, EX94 08826734, and the hospitality of the Institut d'Astrophysique de Paris, where the writing of this paper was finished. The Isaac Newton and Jacobus Kaptein Telescopes are operated on the island of La Palma by the Royal Greenwich Observatory in the Spanish Observatorio del Roque de los Muchachos of the Instituto de Astrofísica de Canarias. This research has made use of the NASA/IPAC EXTRAGALACTIC DATABASE (NED) which is operated by the Jet Propulsion Laboratory, Caltech, under contract with the National Aeronautics and Space Administration.

\section{References}

Afanas'ev V.L., Burenkov A.N., Zasov A.V., Sil'chenko O.K., 1991, Sov. Astron. 35, 574

Andredakis Y.C., Sanders R.H., 1994, MNRAS 267, 283

Arkhipova V.P., Zasov A.V., Noskova R.I., 1987a, Sov. Astron. 31,2

Arkhipova V.P., Zasov A.V., Noskova R.I., Sil'chenko O.K., 1987b, Sov. Astron. 31, 6

Arp H.C., 1966, in "Atlas of Peculiar Galaxies", California Institute of Technology, California 
Arsenault R., 1989, A\&A 217, 66

Athanassoula E., 1984, Phys. Rep. 114, 319

Baesi-Pillastrini G.C., 1987, A\&A 1972, 375

Barnes J.E., Hernquist L., 1992, ARA\&A 30, 705

Bernlhor K., 1993, A\&A 268, 25

Boroson T., 1981, ApJS 46, 177

Burstein D., Heiles C., 1984, ApJS 54, 33

Bushouse H.A., 1987, ApJ 320, 49

Campos-Aguilar A., Moles M., 1991, A\&A 241, 358

Campos-Aguilar A., Moles M., Masegosa J., 1993, AJ 106, 1784

de Vaucouleurs G., 1948, Ann. Astrophys. 11, 247

de Vaucouleurs G., de Vaucouleurs A., Corwin H.G., Buta R., Paturel G., Fouqué P., 1991, Third Reference Catalogue of Bright Galaxies. Springer, New York (RC3)

Elmegreen D.M., Elmegreen B.G., 1982, MNRAS 201, 1021

Elmegreen D.M., Elmegreen B.G., 1987, ApJ 314, 3

Elmegreen D.M., Elmegreen B.G., Bellin A.D., 1990, ApJ 364, 415

Evans R., 1994, MNRAS 266, 511

Freeman K.C., 1970, ApJ 160, 811

Gallagher J.S., Hunter D.A., Tutukov A.V., 1984, ApJ 284, 544

Ho L.C, Filippenko A.V., Sargent W.L.W., 1995, ApJS 98, 477

Huchra J., Davis M., Latham D., Tonry J., 1983, ApJS 52, 89

Joseph R.D., Wright G.S., 1985, MNRAS 214, 87

Karachentsev I.D., 1972, Soob. Sb. Asrt. Observatory Akad, Nauk, 7

Karachentsev I.D., 1980, ApJS 44, 137

Karachentsev I.D., 1987, Binary Galaxies, Moscow, Nauka

Karachentsev I.D., Karachentseva V.E., 1974, Astr. Zh. 51, 724

Keel W.C., Kennicutt R.C., Hummel E., van der Hulst J.M., 1985, AJ 90, 708

Kennicutt R.C., 1983, AJ 272, 54

Kennicutt R.C., Kent S.M., 1983, AJ 88, 1094

Kennicutt R.C., Keel W.C., van der Hulst J.M., Hummel E., Roettiger K.A., 1987, AJ 320, 49

Kent S.M., 1985, ApJS 59, 115

Kent S.M., 1986, AJ 91, 1301

Knapen J.H., van der Kruit P.C., 1991, A\&A 248, 57

Kraan-Korteweg R.C., 1986, A\&AS 66, 255

Landoldt U.E., 1983, AJ 88, 439

Larson R.B., Tinsley B.M., 1978, ApJ 219, 46

Larson R.B., 1992, en "Physics of Nearby Galaxies: Nature or Nurture?". In: Thuan T.X., Balkowski C. \& Thranh Van
J.-T. (eds.). Editions Frontières, France

Laurikainen E., Moles M., 1988, AJ 96, 470

Laurikainen E., Moles M., 1989, AJ 345, 176

Márquez I., 1994, Thesis, Universidad de Granada

Márquez I., Moles M., 1993, AJ 105, 2090

Márquez I., Moles M., 1994, AJ 108, 90

Márquez I., Moles M., Masegosa J., 1996, A\&A 310, 401

Moffat A.F.J., 1969, A\&A 3, 455

Moles M., García-Pelayo J.M., 1985, Memoria Técnica del Instituto Geográfico Nacional, Madrid

Moles M., del Olmo A., Perea J., Masegosa J., Márquez I, Costa V., 1994a, A\&A 285, 404

Moles M., Márquez I., Masegosa J., del Olmo A., Perea J., Arp H., 1994b, ApJ 432, 135

Moles M., Márquez I., Pérez E., 1995, ApJ 438, 604

Neckel T.H., Chini R., 1980, A\&AS 39, 411

Nilson P., 1973, Upsala General Catalogue of Galaxies, Nova Acta Reginae Societatis Scientiarum Upsaliensis, Ser V: A, Vol. 1, Upsala

Noguchi M., 1987, MNRAS 228, 635

Noguchi M., 1990, en "Paired and Interacting Galaxies", Proceedings of the IAU Coll. 124. In: Sulentic J.W., Keel W.C., Telesco C.M. (eds.), NASA-CP3098, p. 711

Peterson Ch.J., Rubin V.C., Ford W.K., Roberts M.S., 1978, ApJ 226, 770

Ohta K., Hamabe M., Wakamatsu K., 1990, ApJ 357, 71

Oke J.B., 1974, ApJS 27, 21

Puerari I., Dottori H.A., 1992, A\&AS 93, 469

Sakhibov F.H., Smirnov M.A., 1990, Astron. Zu. 67, 690

Sargent A., Scoville N., 1991, ApJ 366, L1

Schweizer F., 1976, ApJS 31, 313

Soares D.S.L., 1989, Thesis, University of Groningen

Sofue Y., Yoshida S., Aoki T., et al., 1994, Publ. Astron. Soc. Japan 46, 1

Tonry J.L., Davis M., 1979, AJ 84, 1511

Toomre A., Toomre J., 1972, ApJ 179, 623

Toomre A., 1974, in "The Formation and Dynamics of Galaxies". In: Slakeshaft (ed.). Reidel Pub.

Turner E.L., 1976, ApJ 208, 20

Véron-Cetty M.-P., Véron P., 1993, A Catalogue of Quasars and Active Nuclei, 5th Edition. ESO Scientific Report

Vorontsov-Velyaminov B.A., 1959, "Atlas and Catalogue of Interacting Galaxies". Sternberg Astron. Inst. Moscow 

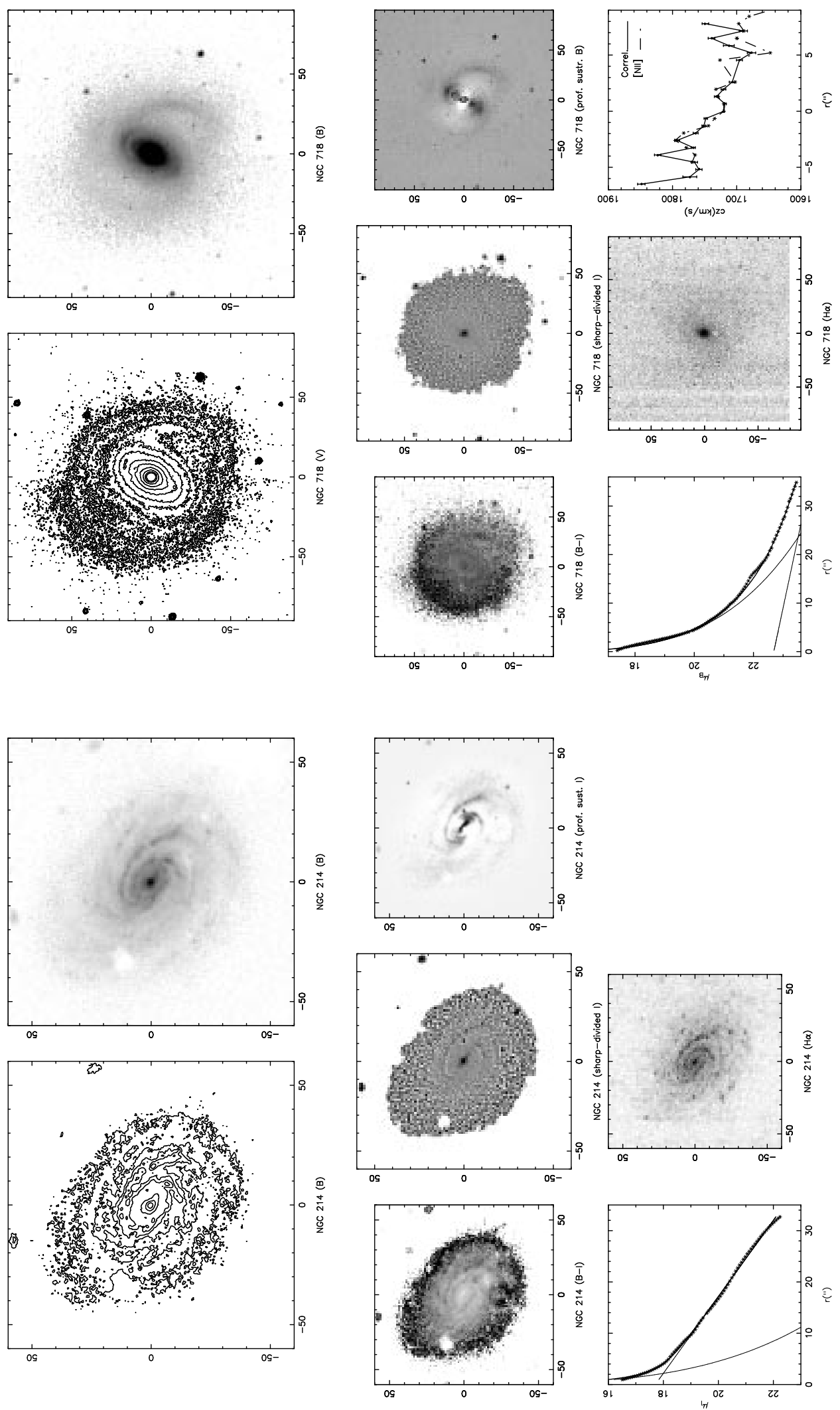

Fig. 2. North is at the top, East to the left. Coordinates are in arcseconds from the center. a) Isophotal image. b) B greyscale image. c) Greyscale $(B-V)$ color index image. Darker is bluer. d) Sharp-divided $B$ image (see text). e) Residual image resulting from the subtraction of a synthetic image from the original one. The parameters used to construct the artificial image are those from the isophotal decomposition together with the galactic orientation in the sky. f) Surface brightness profile. Isophotal magnitudes are plotted versus equivalent radii. A fit by bulge and disk components is also presented (see text). $\mathrm{g}$ ) $\mathrm{H} \alpha$ (continuum subtracted) greyscale image. h) Rotation curve as derived from long slit data 

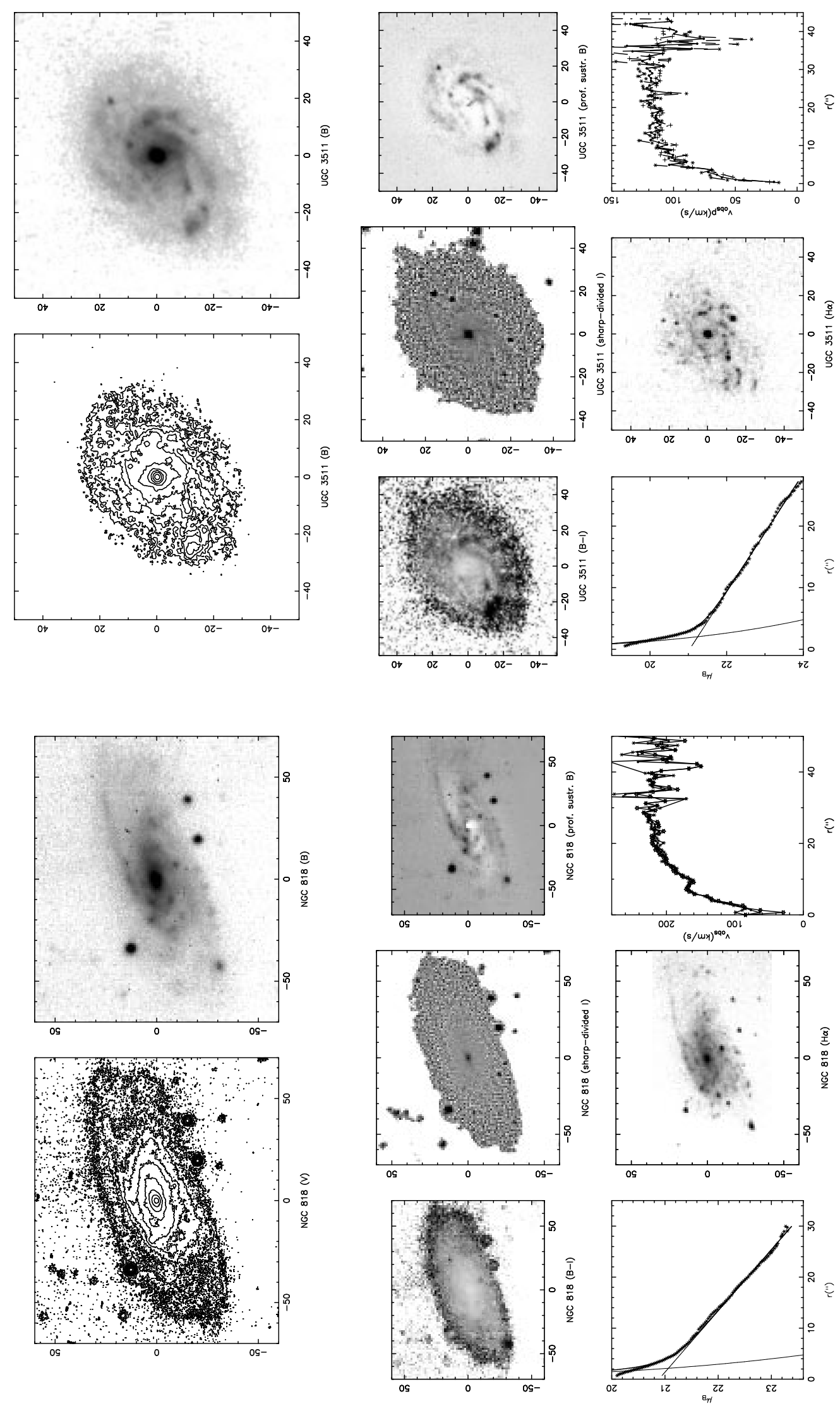

Fig. 2. continued 

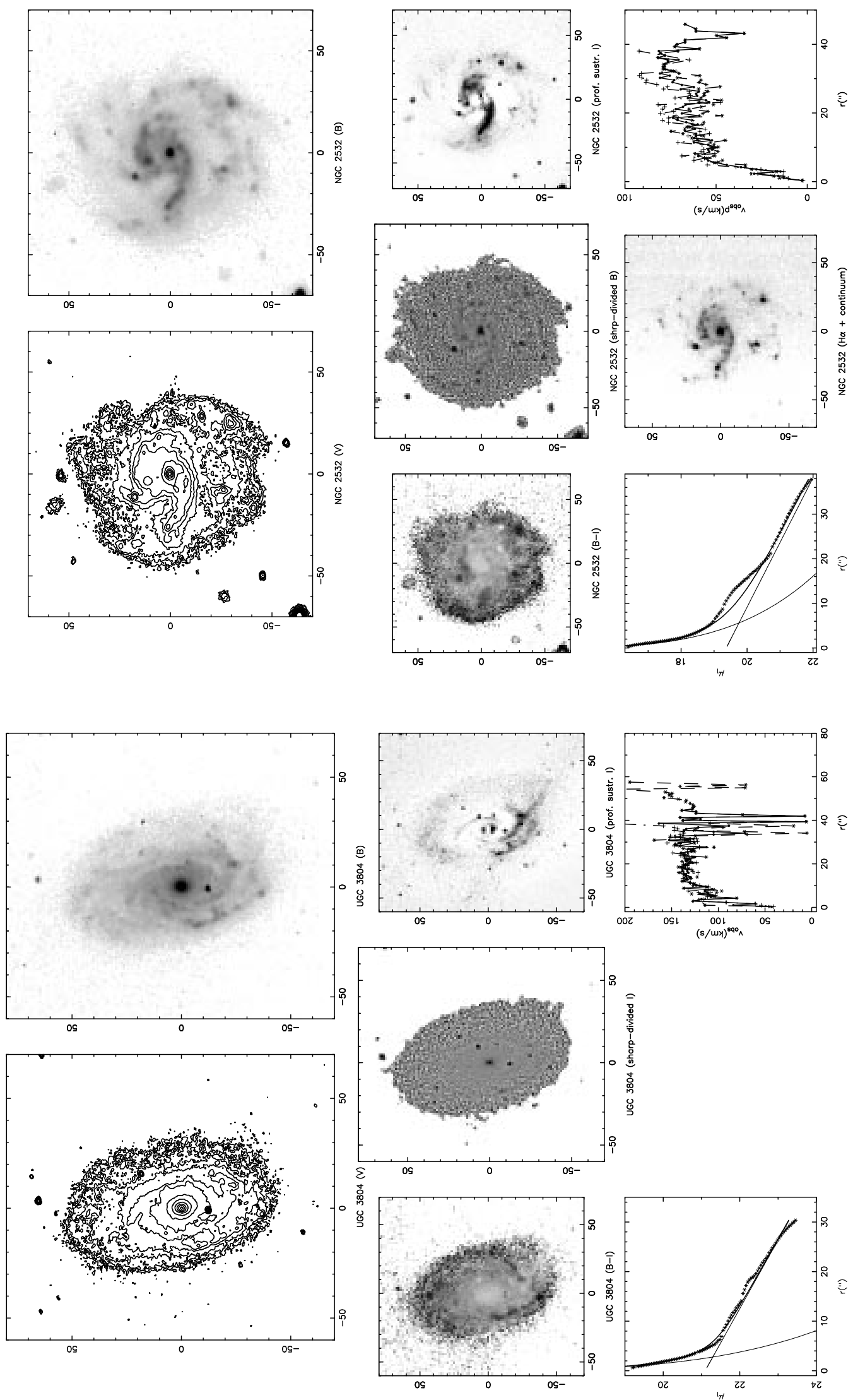

Fig. 2. continued 

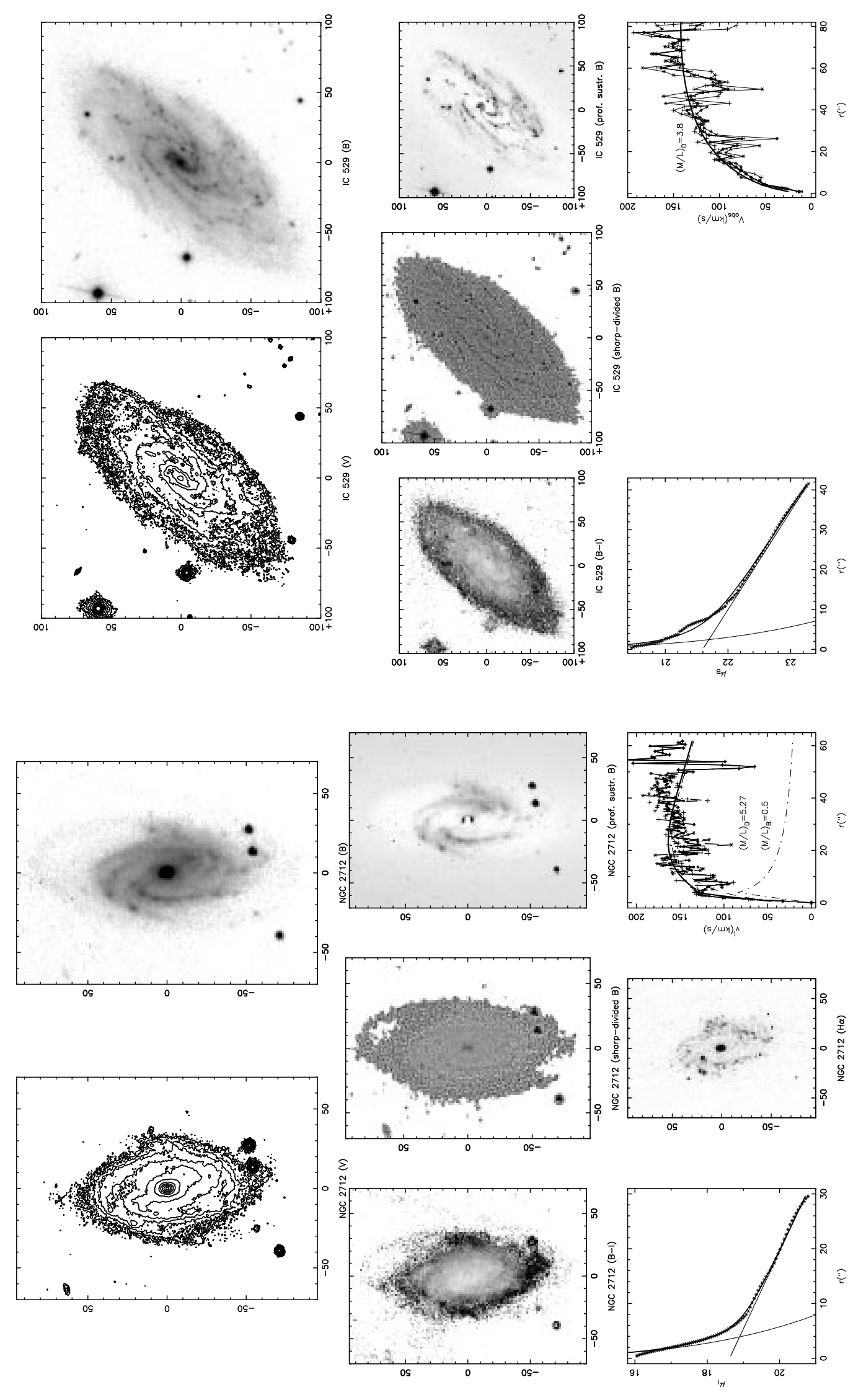

Fig. 2. continued 

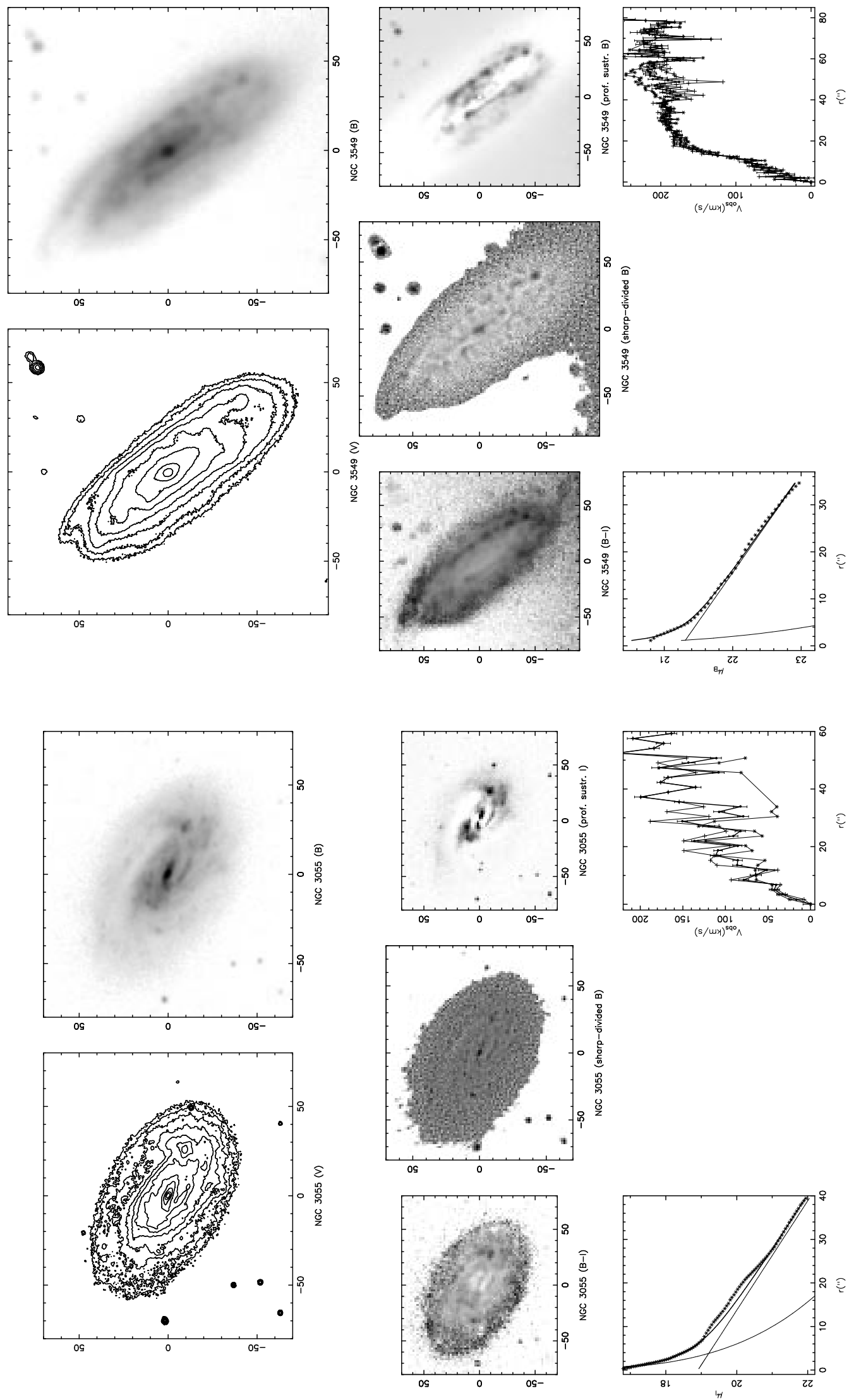

Fig. 2. continued 

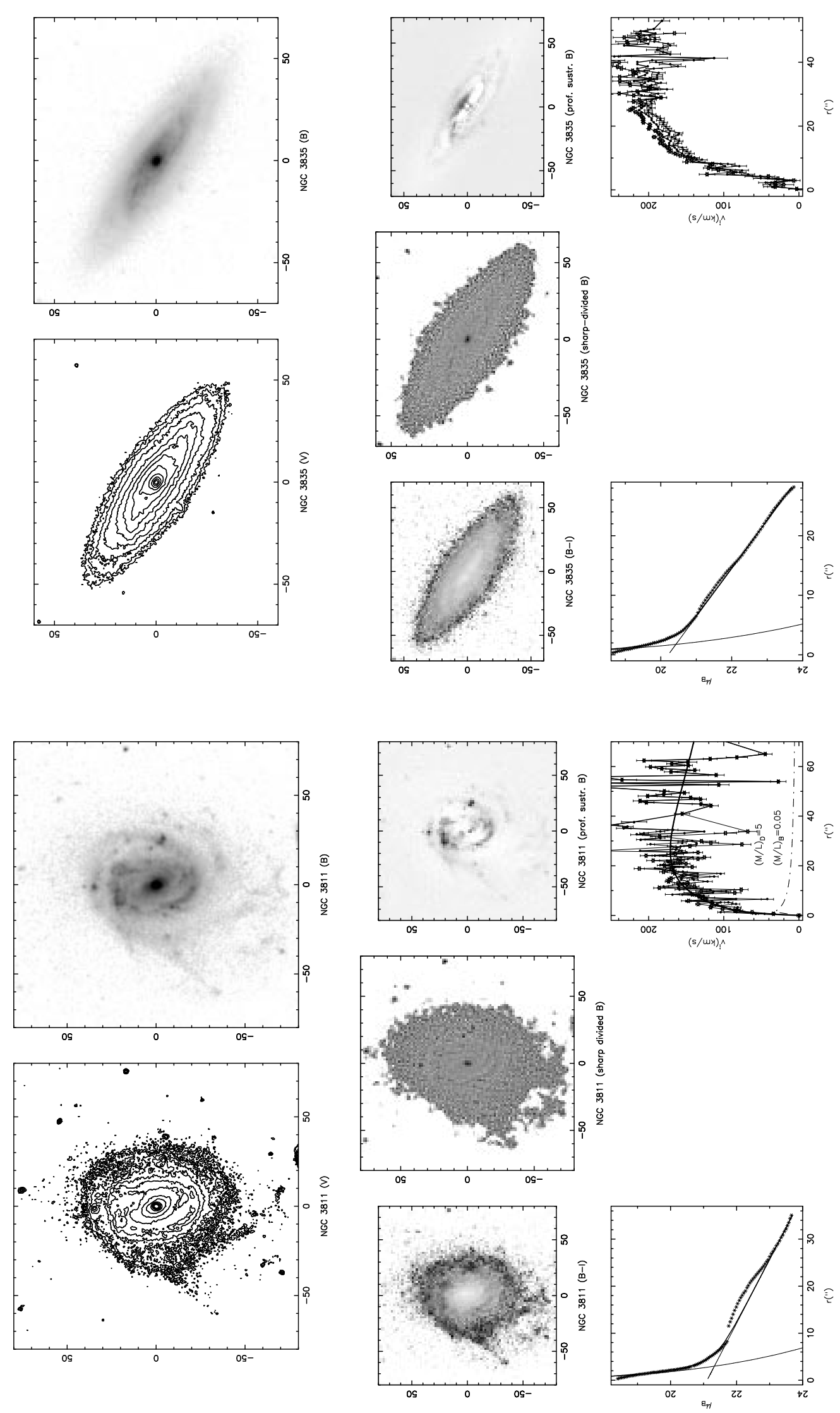

Fig. 2. continued 

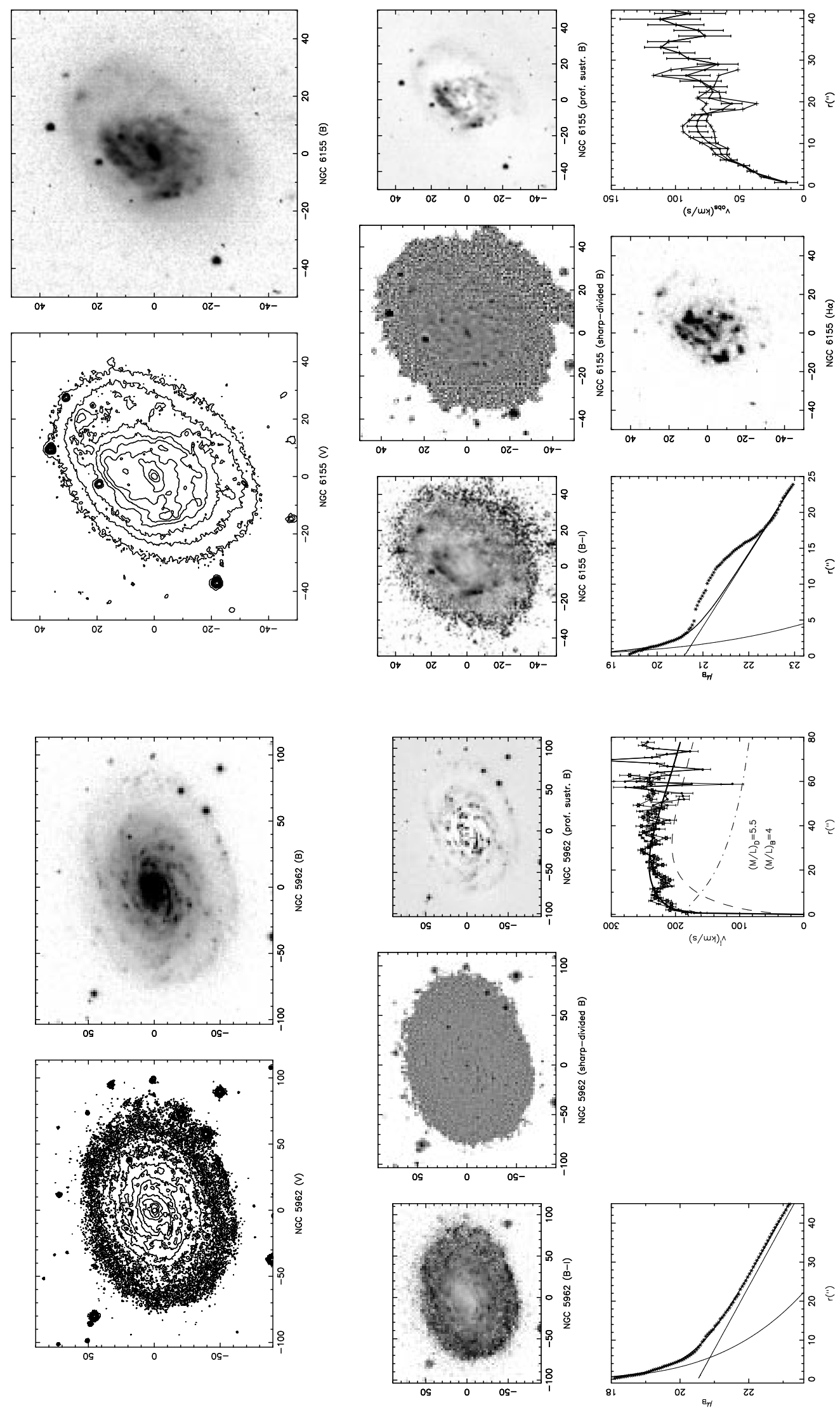

Fig. 2. continued 

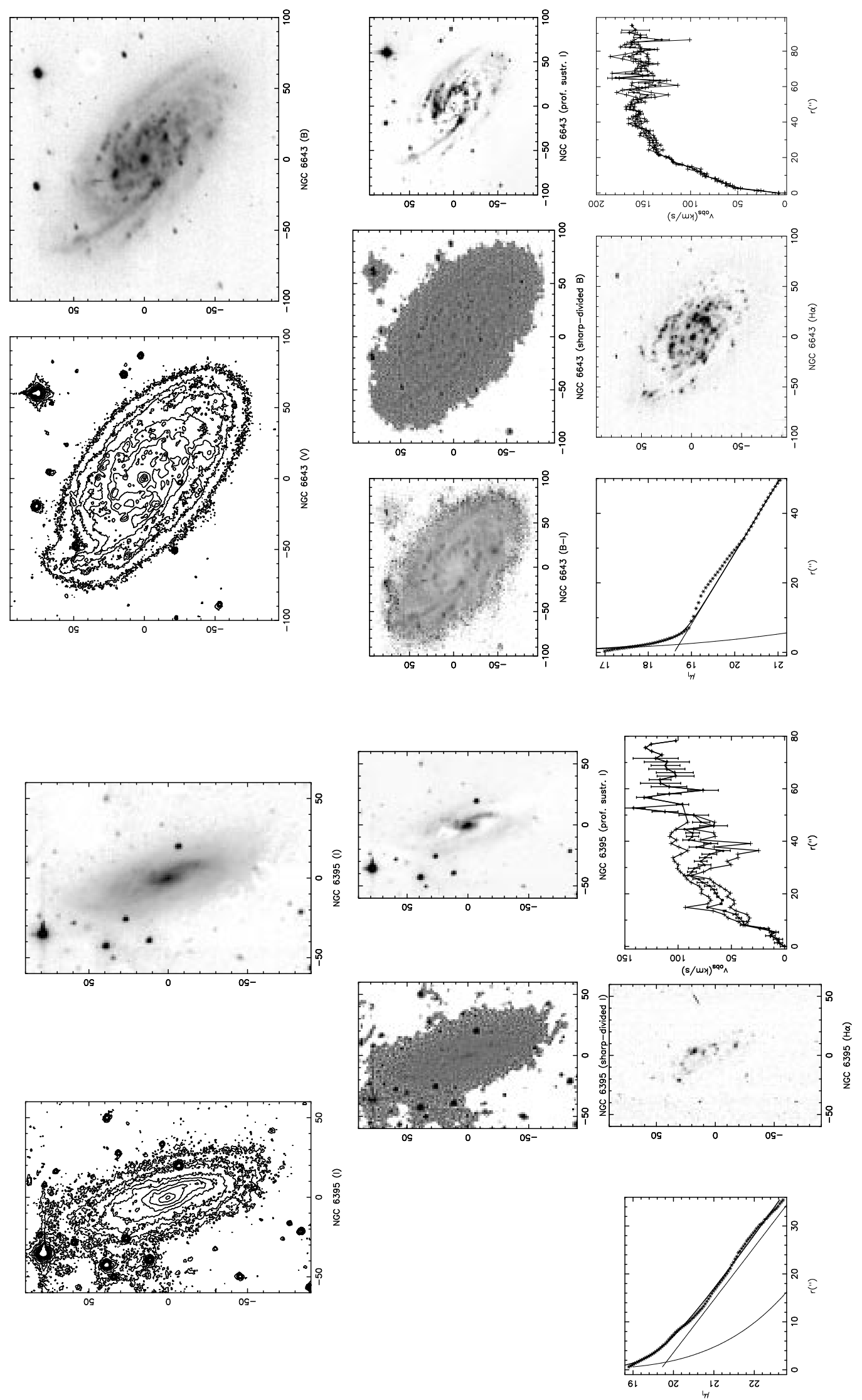

Fig. 2. continued 

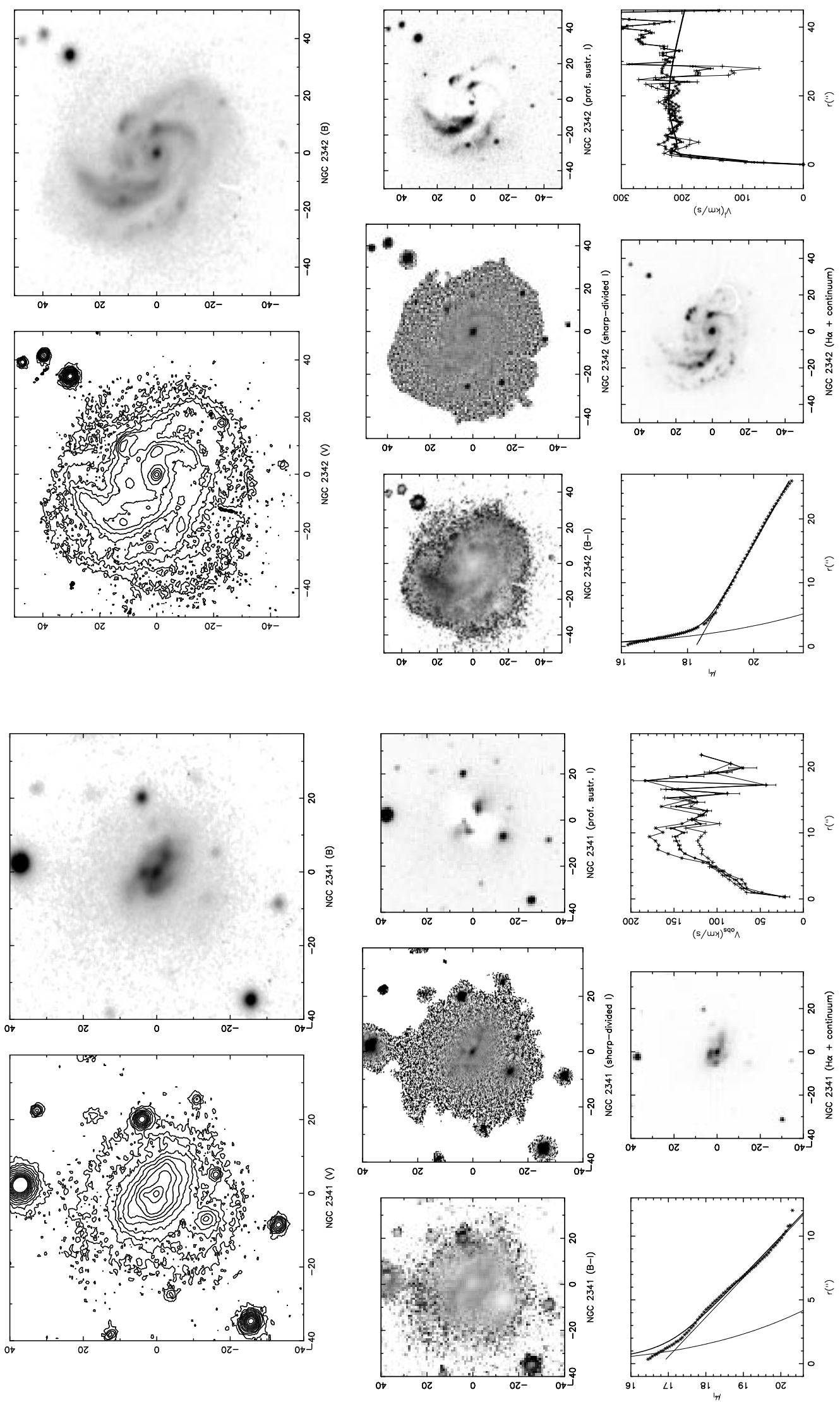

Fig. 2. continued 

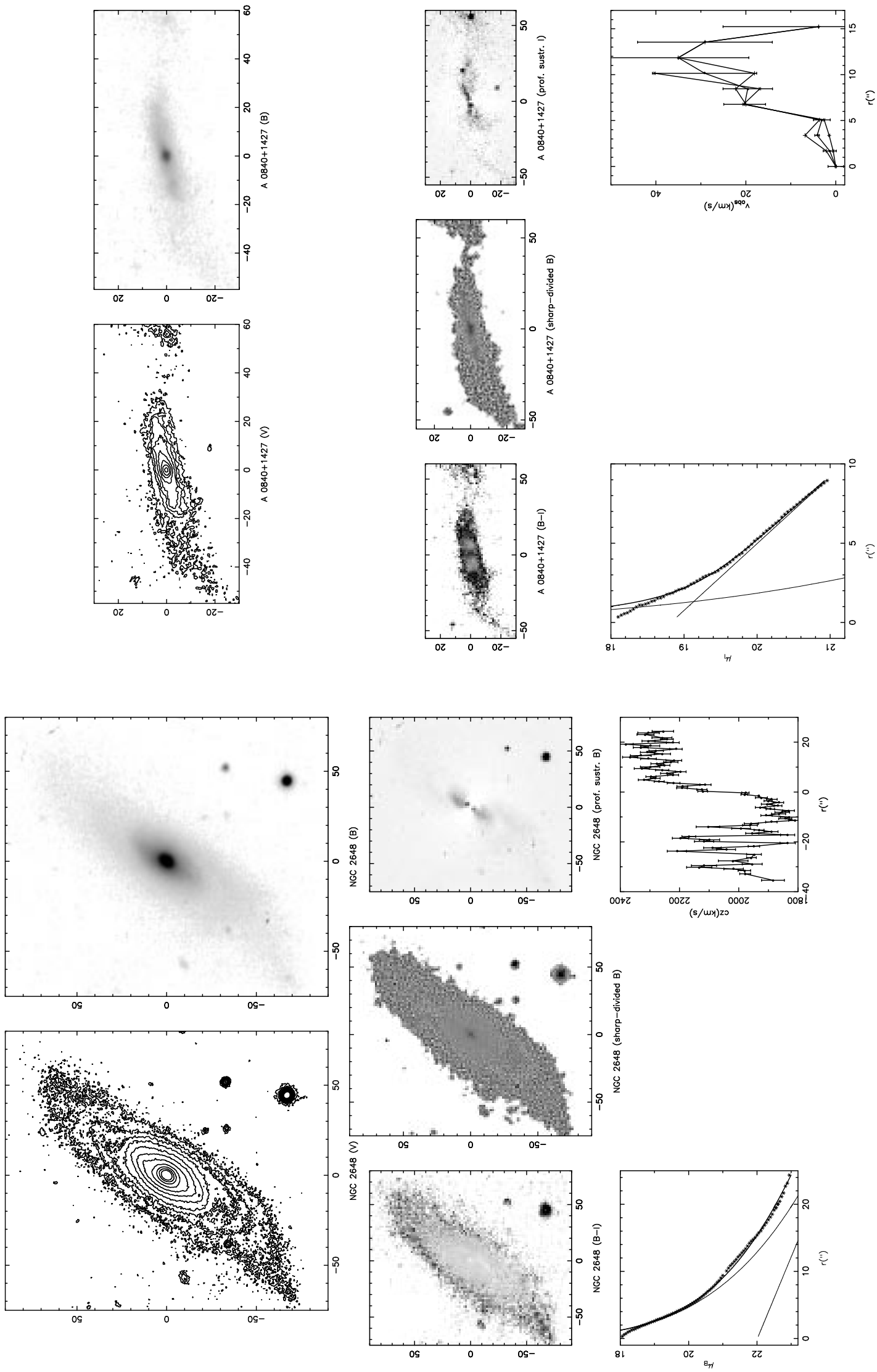

Fig. 2. continued 

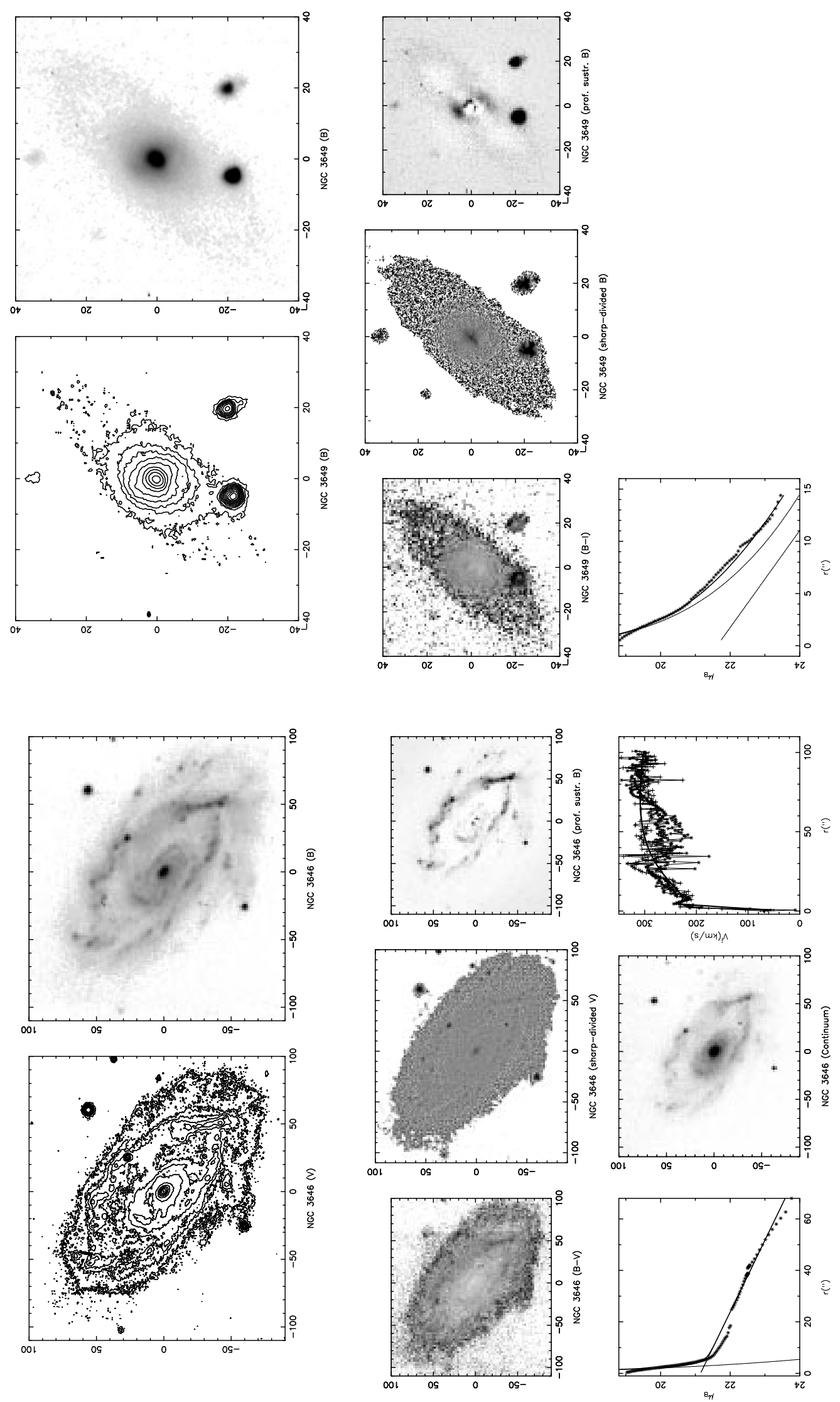

Fig. 2. continued 

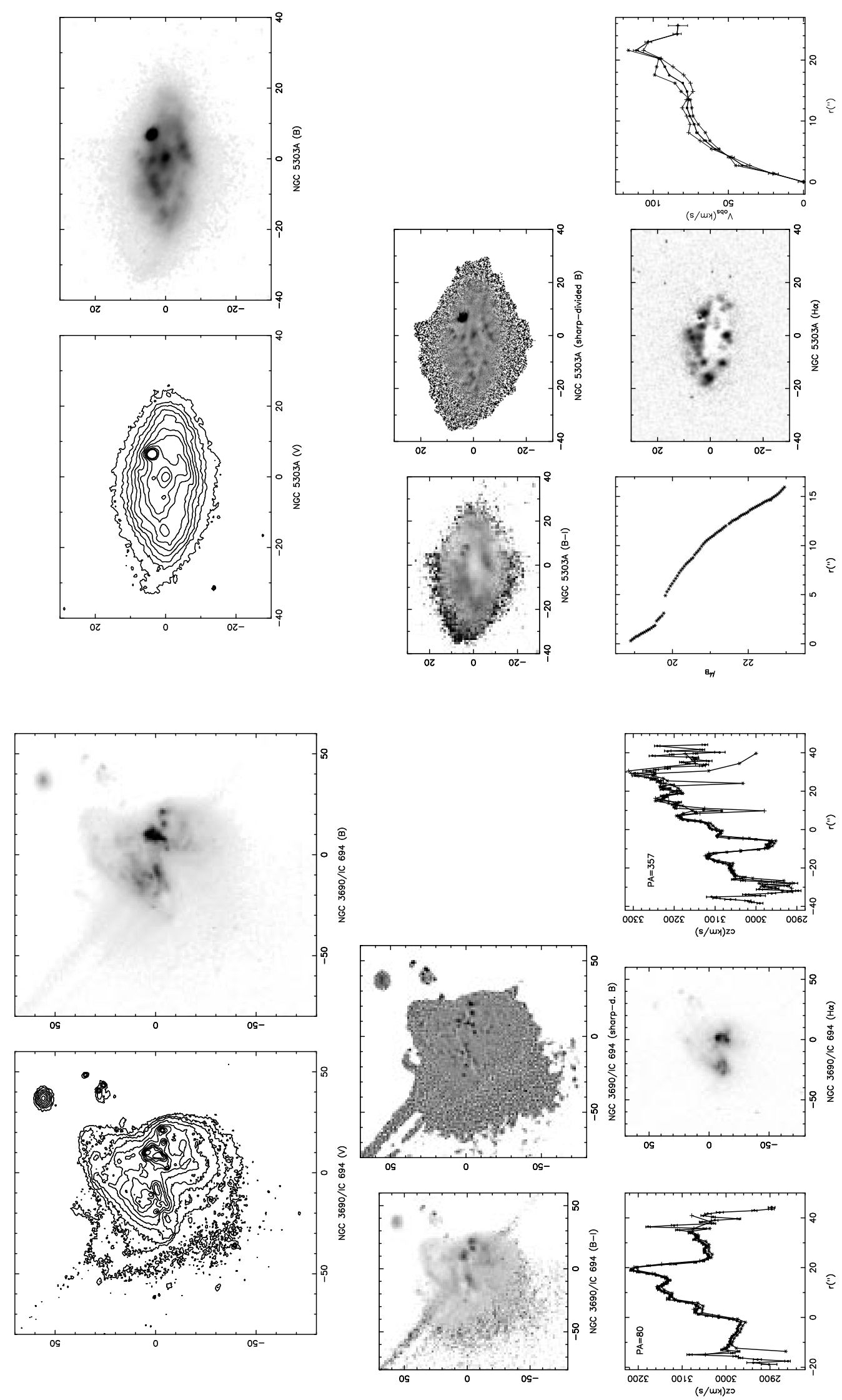

Fig. 2. continued 

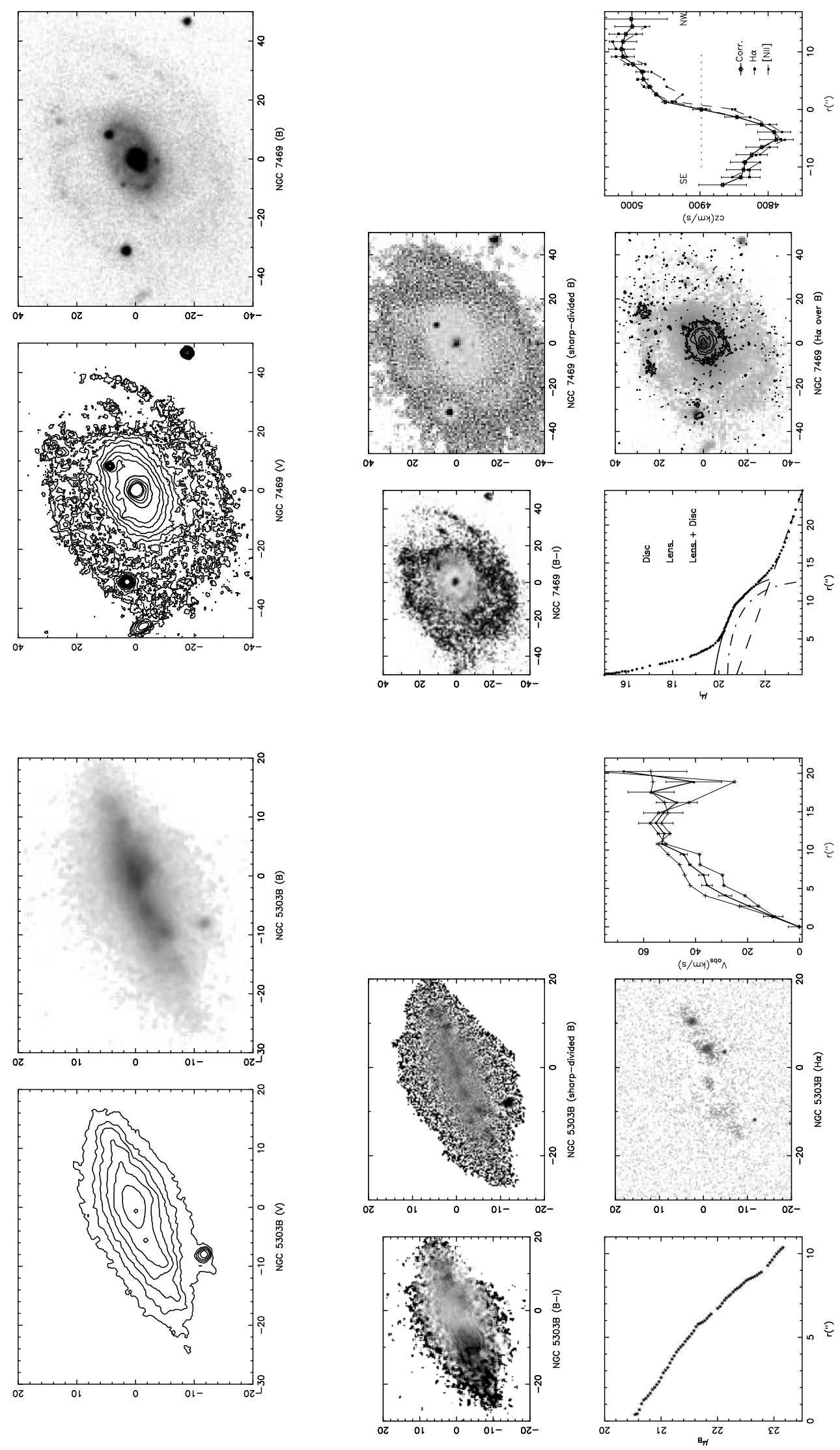

Fig. 2. continued 

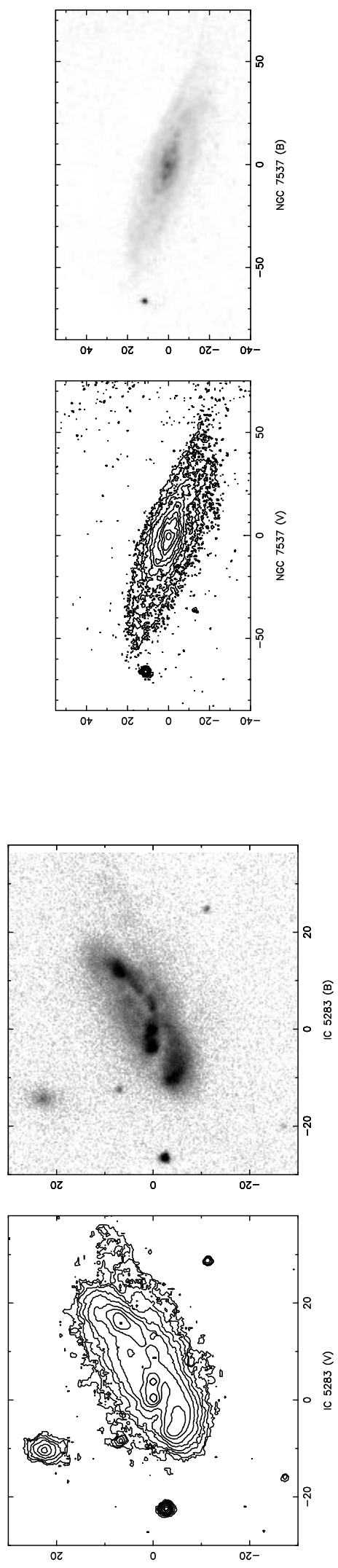
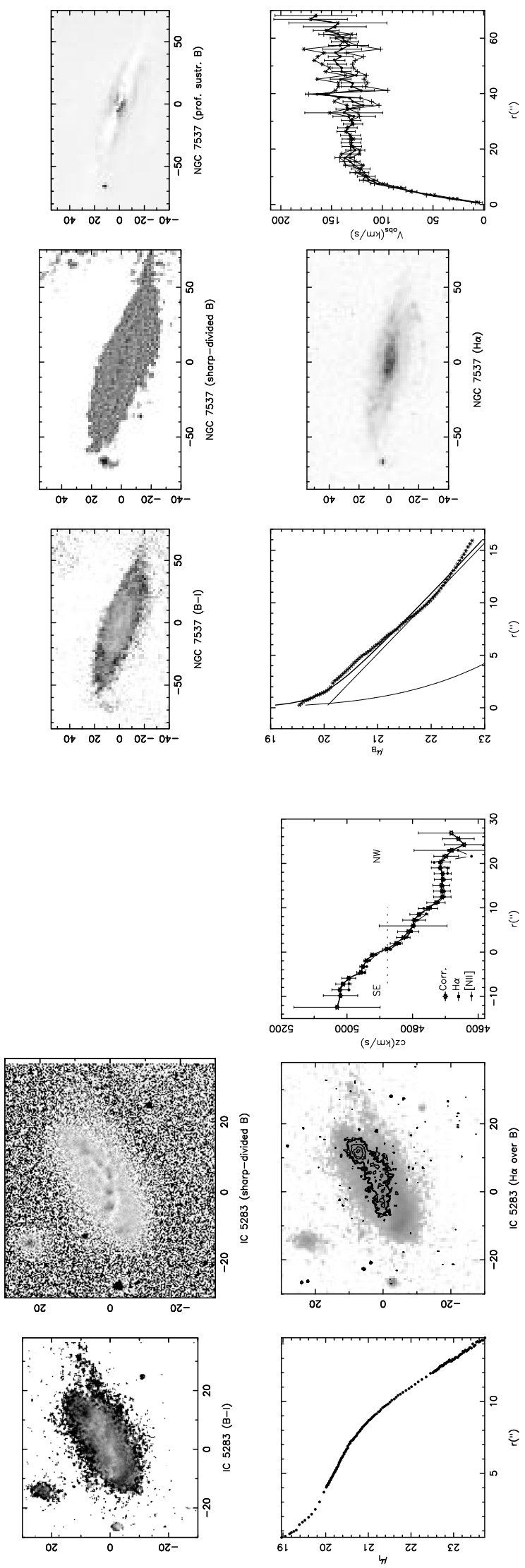

Fig. 2. continued 

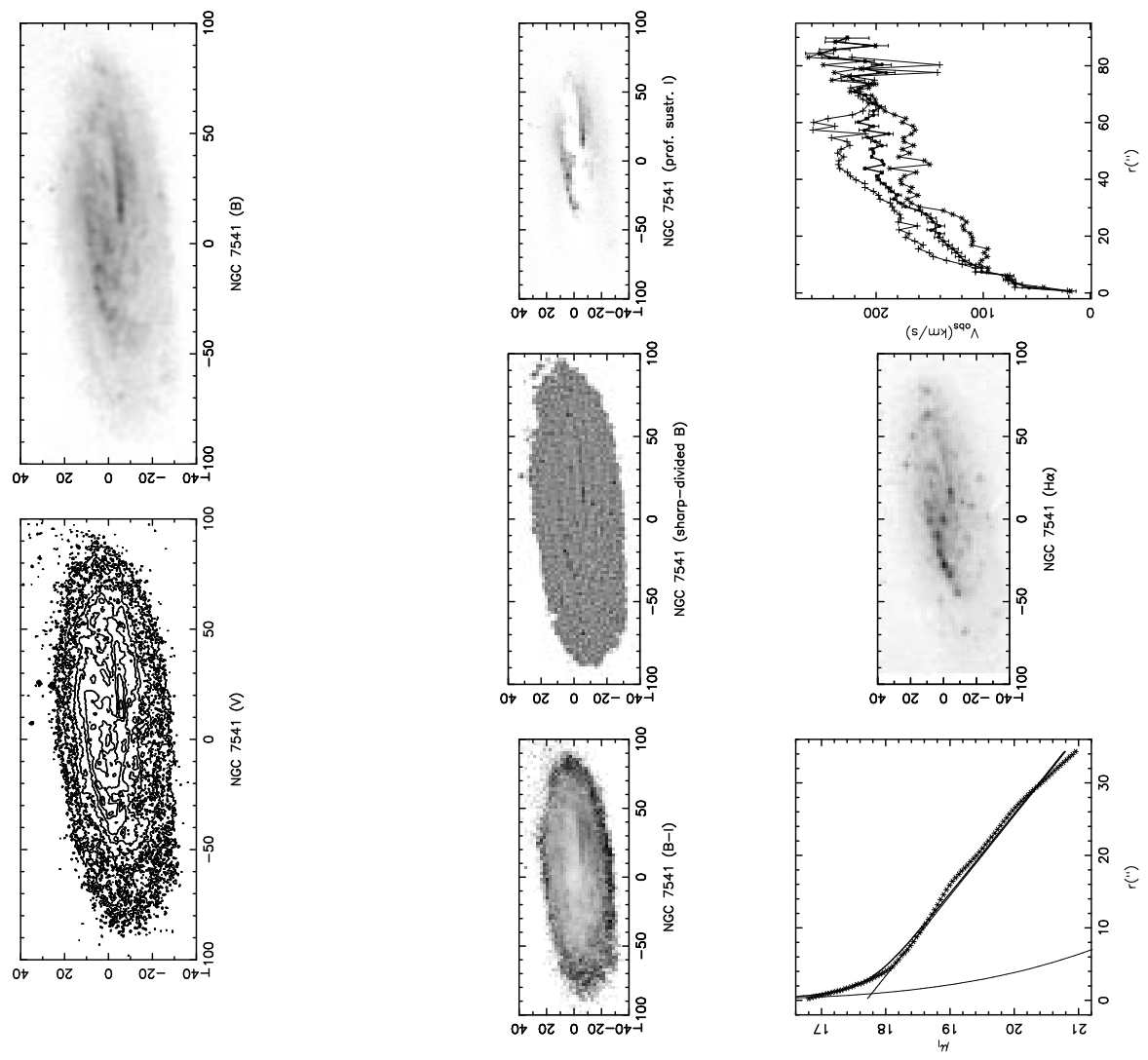

Fig. 2. continued 